\title{
Photoactivable heterocyclic cages in a comparative release study of butyric acid as a model drug
}

\author{
Ana M. Piloto, ${ }^{a}$ Graham Hungerford, ${ }^{b}$ Jens U. Sutter, ${ }^{c}$ Susana P. G. Costa ${ }^{a}$ and M. Sameiro T. \\ Gonçalves $^{\mathrm{a}}$ \\ ${ }^{a}$ Centro de Química, Universidade do Minho, Campus de Gualtar, 4710-057 Braga, Portugal \\ ${ }^{\mathrm{b}}$ HORIBA Jobin Yvon IBH Ltd, 45 Finnieston Street, Glasgow G3 8JU, UK \\ ${ }^{\mathrm{c}}$ Photophysics Group, Centre for Molecular Nanometrology, Department of Physics, Scottish Universities \\ Physics Alliance, University of Strathclyde, Glasgow G4 ONG, UK
}

\begin{abstract}
Aiming at the improvement of the photorelease of butyric acid - a model carboxylic acid drug, a set of heteroaromatic compounds based on acridine, naphtho[2,1-b]pyran, $3 H$-benzopyran fused julolidine and thioxo-naphtho[2,1-b]pyran were evaluated as benzyl-type phototriggers, in comparison with the well-known $o$-nitrobenzyl group. The corresponding ester cages were irradiated in a photochemical reactor at 254,300, 350 and $419 \mathrm{~nm}$, in two solvent systems (methanol or acetonitrile in 80:20 mixtures with HEPES buffer). Photolysis studies showed that, for some of the cages, the release of the active molecule occurred with short irradiation times at $419 \mathrm{~nm}$. Timeresolved fluorescence was used to elucidate their photophysical properties and determine the decay kinetics. Studies were also carried out to assess the suitability of using two-photon excitation to address these compounds, which is advantageous if their use in biological systems is to be considered.
\end{abstract}

\section{Keywords}

Prodrugs; Butyric acid; Coumarin; Acridine; $o$-Nitrobenzyl group; Phototriggers.

\section{Introduction}

The use of several drugs in clinical practice is dependent on the improvement of their therapeutic effects, bioavailability, physicochemical properties and the minimizing of other undesirable side effects. Prodrugs, pharmacologically latent derivatives of active agents, can be designed to undergo 
activation through a specific stimulus. In addition to chemical and/or enzymatic triggers, light is an appealing tool to use for conversion of prodrugs to active agents in a spatially and temporally controlled manner [1-8]. Butyric acid, a saturated unbranched monocarboxylic acid, is one of the short-chain fatty acids. The effects of butyric acid include the disruption of cell proliferation and induction of apoptosis; modification of cell morphology and alteration of gene expression [9]. The presence of carboxylic acids or other ionisable polar groups in drugs can result in poor absorption from the gastrointestinal tract owing to lipophilicity/solubility issues.

Light triggerable benzyl or heterocyclic benzyl esters represent a large family of photolabile protecting groups. $o$-Nitrobenzylic derivatives have been widely used in various applications, as they combine a satisfactory photosensitivity with a stability for handling and synthesis purposes [10-12]. Nevertheless, $o$-nitrobenzyl cages exhibit some limitations, since the wavelength of excitation required for the uncaging is not necessarily the most suitable for bioapplications. The search for protecting groups with improved photochemical properties and even displaying fluorescence has motivated the incorporation of heterocyclic moieties in their design, with coumarinyl methyl groups as relevant examples. It is possible to find a wide range of derivatives possessing different combinations of substituents and/or fusion ring units for the release of various active molecules [1315]. Recently, acridinyl methyl esters have also proved to possess the required photosensitivity for the release of carboxylic acid compounds [16].

Considering the know-how of the authors in the field of fluorescent photoactivable molecules based on aromatic and heteroaromatic skeletons for the release of bioanalytes [17-24], in connection with the interest in the development of alternative light sensitive prodrugs, and following the previous work regarding butyric acid, a new set of oxygen and nitrogen heterocyclic cages were synthesised. The use of these heterocyclic moieties can produce longer maximum wavelengths of absorption, allowing the photorelease of butyric acid at longer wavelengths, not detrimental to bioapplications. It also opens the way to use two-photon excitation (TPE), where if sufficient photon flux is present two longer wavelength photons can be used to excite a sample. This is advantageous as it only occurs in a fentolitre volume and the longer wavelength photons are less likely to interact with biological material. Thus, the present work evaluates the behaviour of (acridin-9-yl)methyl, (5-methoxy-3-oxo$3 H$-naphtho[2,1-b]pyran-1-yl)methyl, (8-methoxy-3-oxo-3H-naphtho[2,1-b]pyran-1-yl)methyl, and [11-oxo-2,3,5,6,7,11-hexahydro- $1 H$-pyrano[2,3-f]pyrido[3,2,1-ij]quinolin-9-yl]methyl groups, in comparison with the well-known $o$-nitrobenzyl group in the light-induced release of butyric acid. Additionally, bearing in mind that the replacement of a carbonyl by a thiocarbonyl group results in 
an improvement in the photolytic release [23,24], thionated groups; namely (5-methoxy-3-thioxo3H-naphtho[2,1-b]pyran-1-yl)methyl， (8-methoxy-3-thioxo-3H-naphtho[2,1-b]pyran-1-yl)methyl, and (9-methoxy-3-thioxo-3H-naphtho[2,1-b]pyran-1-yl)methyl were tested. The ester cages, in two solvent systems (methanol or acetonitrile in 80:20 mixtures with HEPES buffer), were irradiated at $254,300,350$ and $419 \mathrm{~nm}$ in a photochemical reactor. The fact that the groups exhibit fluorescence enabled time-resolved fluorescence measurements to be employed to elucidate their photophysical properties and determine the decay kinetics, along with their suitability for two-photon excitation. This last point can be important if their use in biological systems is to be considered

\section{Experimental}

\subsection{Synthesis general}

All melting points were measured on a Stuart SMP3 melting point apparatus. TLC analyses were carried out on $0.25 \mathrm{~mm}$ thick precoated silica plates (Merck Fertigplatten Kieselgel $60 \mathrm{~F}_{254}$ ) and spots were visualised under UV light. Chromatography on silica gel was carried out on Merck Kieselgel (230-240 mesh). IR spectra were determined on a BOMEM MB 104 spectrophotometer. UV/visible absorption spectra $(200-700 \mathrm{~nm})$ were obtained using a Shimadzu UV/2501PC spectrophotometer. NMR spectra were obtained on a Bruker Avance III 400 at an operating frequency of $400 \mathrm{MHz}$ for ${ }^{1} \mathrm{H}$ and $100.6 \mathrm{MHz}$ for ${ }^{13} \mathrm{C}$ using the solvent peak as internal reference at $25^{\circ} \mathrm{C}$. All chemical shifts are given in ppm using $\delta_{\mathrm{H}} \mathrm{Me}_{4} \mathrm{Si}=0 \mathrm{ppm}$ as reference and $J$ values are given in $\mathrm{Hz}$. Assignments were made by comparison of chemical shifts, peak multiplicities and $J$ values and were supported by spin decoupling-double resonance and bidimensional heteronuclear correlation techniques. Mass spectrometry analyses were performed at the "C.A.C.T.I. - Unidad de Espectrometria de Masas", at University of Vigo, Spain. Fluorescence spectra were collected using a FluoroMax-4 spectrofluorometer. All reagents were used as received. Compounds 5, $\mathbf{6}$ and $\mathbf{1 1}$ were synthesised as previously reported $[20,25,26]$.

2.2. Synthesis of 1-chloromethyl-5-methoxy-3-oxo-3H-naphtho[2,1-b]pyran 3. To a solution of 3methoxy-2-naphthol $\left(0.104 \mathrm{~g}, 5.97 \times 10^{-4} \mathrm{~mol}\right)$ in $70 \%$ aqueous sulphuric acid $(5 \mathrm{~mL})$, ethyl 4chloro-3-oxobutanoate $\left(0.089 \mathrm{~mL}, 6.57 \times 10^{-4} \mathrm{~mol}\right)$ was added. The reaction was followed by TLC (ethyl acetate $/ n$-hexane, 1:4), and stirred at room temperature for $96 \mathrm{~h}$. The mixture was poured into ice water and stirred for $2 \mathrm{~h}$ to give a fine pale precipitate. The solid was collected by filtration, washed with cold water, dried and purified by column chromatography, using ethyl acetate/ light 
petroleum as eluent, with mixtures of increasing polarity. Compound $\mathbf{3}$ was obtained as pale brown solid (0.012 g, 7\%). Mp 169.5-171.8 ${ }^{\circ} \mathrm{C} . R_{\mathrm{f}}=0.50$ (ethyl acetate/n-hexane, $\left.1: 4\right) .{ }^{1} \mathrm{H}$ NMR (400 MHz, $\left.\mathrm{CDCl}_{3}\right): \delta_{\mathrm{H}}=4.03\left(\mathrm{~s}, 3 \mathrm{H}, \mathrm{OCH}_{3}\right), 5.05\left(\mathrm{~s}, 2 \mathrm{H}, \mathrm{CH}_{2}\right), 6.75(\mathrm{~s}, 1 \mathrm{H}, \mathrm{H}-2), 7.36(\mathrm{~s}, 1 \mathrm{H}, \mathrm{H}-6), 7.54-$ 7.57 (m, 2 H, H-8 and H-9), 7.82-7.84 (m, $1 \mathrm{H}, \mathrm{H}-7), 8.30-8.32$ (m, $1 \mathrm{H}, \mathrm{H}-10) .{ }^{13} \mathrm{C}$ NMR (100.6 $\left.\mathrm{MHz}, \mathrm{CDCl}_{3}\right): \delta_{\mathrm{C}}=45.79\left(\mathrm{CH}_{2}\right), 56.16\left(\mathrm{OCH}_{3}\right), 111.17(\mathrm{C}-6), 113.51(\mathrm{C}-4 \mathrm{~b}), 117.74(\mathrm{C}-2), 123.69$ (C-6b), 124.71 (C-10), 125.95 (C-9), 126.17 (C-8), 128.46 (C-7), 131.25 (C-6a), 146.75 (C-5), 147.21 (C-4a), 151.33 (C-1), 159.22 (C-3). IR (KBr 1\%): v = 1728, 1627, 1599, 1556, 1513, 1486, 1462, 1439, 1422, 1378, 1348, 1325, 1264, 1217, 1168, 1140, 1122, 1046, 1024, 1001, 938, 896, 866, 844, 778, 737, $704 \mathrm{~cm}^{-1}$. HRMS (ESI) for $\mathrm{C}_{15} \mathrm{H}_{12}{ }^{37} \mathrm{ClO}_{3}\left[\mathrm{M}^{+}+\mathrm{H}\right]$ : calculated 277.04456, found 277.04478; for $\mathrm{C}_{15} \mathrm{H}_{12}{ }^{35} \mathrm{ClO}_{3}\left[\mathrm{M}^{+}+\mathrm{H}\right]$ : calculated 275.04756, found 275.04770.

2.3. Synthesis of 1-chloromethyl-8-methoxy-3-oxo-3H-naphtho[2,1-b]pyran 4.To a solution of 6methoxy-2-naphthol $\left(0.248 \mathrm{~g}, 1.42 \times 10^{-3} \mathrm{~mol}\right)$ in $70 \%$ aqueous sulphuric acid $(5 \mathrm{~mL})$, ethyl 4 chloro-3-oxobutanoate $\left(0.288 \mathrm{~mL}, 2.13 \times 10^{-3} \mathrm{~mol}\right)$ was added. The reaction was followed by TLC (ethyl acetate/ $n$-hexane, 1:4), and stirred at room temperature for $72 \mathrm{~h}$. The mixture was poured into ice water and stirred for $2 \mathrm{~h}$ to give a fine pale precipitate. The solid was collected by filtration, washed with cold water, dried and purified by column chromatography, using ethyl acetate/light petroleum as eluent, with mixtures of increasing polarity. Compound $\mathbf{4}$ was obtained as pale yellow solid (0.145 g, 37\%). Mp 207.3-209.9 ${ }^{\circ} \mathrm{C} . R_{\mathrm{f}}=0.50$ (ethyl acetate/n-hexane, 1:4). ${ }^{1} \mathrm{H}$ NMR (400 $\left.\mathrm{MHz}, \mathrm{CDCl}_{3}\right): \delta_{\mathrm{H}}=3.97\left(\mathrm{~s}, 3 \mathrm{H}, \mathrm{OCH}_{3}\right), 5.03\left(\mathrm{~s}, 2 \mathrm{H}, \mathrm{CH}_{2}\right), 6.72(\mathrm{~s}, 1 \mathrm{H}, \mathrm{H}-2), 7.26$ (d, J = 2.8 Hz, 1 H, H-7), 7.36 (dd, $J=9.6$ and $2.8 \mathrm{~Hz}, 1 \mathrm{H}, \mathrm{H}-9), 7.46$ (d, $J=8.8 \mathrm{~Hz}, 1 \mathrm{H}, \mathrm{H}-5), 7.92$ (d, $J=8.8 \mathrm{~Hz}, 1$ $\mathrm{H}, \mathrm{H}-6), 8.33(\mathrm{~d}, J=9.2 \mathrm{~Hz}, 1 \mathrm{H}, \mathrm{H}-10) .{ }^{13} \mathrm{C} \mathrm{NMR}\left(100.6 \mathrm{MHz}, \mathrm{CDCl}_{3}\right): \delta_{\mathrm{C}}=45.84\left(\mathrm{CH}_{2}\right), 55.41$ $\left(\mathrm{OCH}_{3}\right), 108.61$ (C-7), 112.70 (C-4b), 117.51 (C-2), 118.18 (C-5), 120.09 (C-9), 123.44 (C-6b), 126.36 (C-10), 132.97 (C-6a), 133.16 (C-6), 150.98 (C-1), 153.76 (C-4a), 157.13 (C-8), 160.09 (C3). IR $(\operatorname{KBr} 1 \%): v=1722,1611,1553,1514,1468,1422,1362,1318,1264,1213,1182,1154$, 1118, 1034, 1015, 998, 912, 897, 878, 858, 816, 737, $704 \mathrm{~cm}^{-1}$. HRMS (ESI) for $\mathrm{C}_{15} \mathrm{H}_{12}{ }^{37} \mathrm{ClO}_{3}$ $\left[\mathrm{M}^{+}+\mathrm{H}\right]$ : calculated 277.04456, found 277.04449; for $\mathrm{C}_{15} \mathrm{H}_{12}{ }^{35} \mathrm{ClO}_{3}\left[\mathrm{M}^{+}+\mathrm{H}\right]$ : calculated 275.04756, found 275.04751 .

2.4. Synthesis of 2-nitrobenzyl butyrate, 7. To a solution of butyric acid $\left(0.263 \mathrm{~mL}, 2.88 \times 10^{-3} \mathrm{~mol}\right)$ in dry DMF $(4 \mathrm{~mL})$ at $0{ }^{\circ} \mathrm{C}$, 1-hydroxybenzotriazole (HOBt) $\left(0.072 \mathrm{~g}, 5.33 \times 10^{-4} \mathrm{~mol}\right)$ was added. After stirring for $10 \mathrm{~min}, N, N^{\prime}$-dicyclohexylcarbodiimide (DCC) $\left(0.114 \mathrm{~g}, 5.52 \times 10^{-4} \mathrm{~mol}\right)$ was added, followed by (2-nitrophenyl)methanol $1\left(0.402 \mathrm{~g}, 2.62 \times 10^{-3} \mathrm{~mol}\right)$. The reaction mixture was 
stirred at room temperature for $72 \mathrm{~h}$ and followed by TLC (ethyl acetate/light petroleum, 1:4). The solid was filtered and the residue was evaporated under vacuum. Cold acetone was added and the dicyclohexylurea precipitate was filtered. The solvent was removed by rotary evaporation under reduced pressure and the crude residue was purified by column chromatography using ethyl acetate/light petroleum, with mixtures of increasing polarity as eluent. Compound $\mathbf{7}$ was obtained as an orange oily solid $(0.169 \mathrm{~g}, 29 \%) . R_{\mathrm{f}}=0.88$ (ethyl acetate/light petroleum, 1:4). ${ }^{1} \mathrm{H}$ NMR (400 $\left.\mathrm{MHz}, \mathrm{CDCl}_{3}\right): \delta_{\mathrm{H}}=0.92\left(\mathrm{t}, J=7.6 \mathrm{~Hz}, 3 \mathrm{H}, \mathrm{CH}_{3}-\mathrm{CH}_{2}-\mathrm{CH}_{2}\right.$ ), 1.65 (sext, $J=7.2 \mathrm{~Hz}, 2 \mathrm{H}, \mathrm{CH}_{3}-\mathrm{CH}_{2}-$ $\mathrm{CH}_{2}$ ), $2.36\left(\mathrm{t}, J=7.2 \mathrm{~Hz}, 2 \mathrm{H}, \mathrm{CH}_{3}-\mathrm{CH}_{2}-\mathrm{CH}_{2}\right), 5.47\left(\mathrm{~s}, 2 \mathrm{H}, \mathrm{CH}_{2}\right), 7.44$ (dt, J= 7.4 and $1.6 \mathrm{~Hz}, 1 \mathrm{H}$, H-4), 7.55 (dd, $J=7.6$ and $0.8 \mathrm{~Hz}, 1 \mathrm{H}, \mathrm{H}-6), 7.62$ (dt, $J=7.4$ and $0.8 \mathrm{~Hz}, 1 \mathrm{H}, \mathrm{H}-5), 8.02$ (dd, $J=$ 8.0 and $0.8 \mathrm{~Hz}, 1 \mathrm{H}, \mathrm{H}-3) .{ }^{13} \mathrm{C} \mathrm{NMR}\left(100.6 \mathrm{MHz}, \mathrm{CDCl}_{3}\right): \delta_{\mathrm{C}}=13.43\left(\mathrm{CH}_{3}-\mathrm{CH}_{2}-\mathrm{CH}_{2}\right), 18.19\left(\mathrm{CH}_{3^{-}}\right.$ $\left.\mathrm{CH}_{2}-\mathrm{CH}_{2}\right), 35.80\left(\mathrm{CH}_{3}-\mathrm{CH}_{2}-\mathrm{CH}_{2}\right), 62.54\left(\mathrm{CH}_{2}\right), 124.79$ (C-3), 128.56 (C-4), 128.84 (C-6), 132.06 (C-1), 133.54 (C-5), 147.39 (C-2), 172.80 (C=O). IR (KBr 1\%): v= 2964, 2931, 2851, 1711, 1613, $1574,1525,1476,1445,1434,1366,1338,1305,1251,1187,1144,1085,1037,989,858,792,726$ $\mathrm{cm}^{-1}$. HRMS (ESI) for $\mathrm{C}_{11} \mathrm{H}_{14} \mathrm{NO}_{4}\left[\mathrm{M}^{+}+\mathrm{H}\right]$ : calculated 224.09232, found: 224.09240 .

2.5. Synthesis of (acridin-9-yl)methyl butyrate, 8 . To a solution of 9-(bromomethyl)acridine 2 (0.038 $\left.\mathrm{g}, 1.39 \times 10^{-4} \mathrm{~mol}\right)$ in dry DMF $(3 \mathrm{~mL})$ potassium fluoride $\left(0.073 \mathrm{~g}, 4.17 \times 10^{-4} \mathrm{~mol}\right)$ and butyric acid $\left(0.014 \mathrm{~mL}, 1.52 \times 10^{-4} \mathrm{~mol}\right)$ were added. The reaction was followed by TLC (ethyl acetate/light petroleum, 1:1), and stirred at room temperature for $15 \mathrm{~h}$. The solvent was removed by rotary evaporation under reduced pressure and the crude residue was purified by column chromatography using ethyl acetate/light petroleum, mixtures of increasing polarity as eluent. Compound $\mathbf{8}$ was obtained as a brown oily solid $(0.027 \mathrm{~g}, 69 \%) . R_{\mathrm{f}}=0.48$ (ethyl acetate/light petroleum, 1:1). ${ }^{1} \mathrm{H}$ NMR (400 MHz, $\left.\mathrm{CDCl}_{3}\right): \delta_{\mathrm{H}}=0.89\left(\mathrm{t}, J=7.2 \mathrm{~Hz}, 3 \mathrm{H}, \mathrm{CH}_{3}-\mathrm{CH}_{2}-\mathrm{CH}_{2}\right), 1.63(\mathrm{sext}, J=7.2 \mathrm{~Hz}, 2 \mathrm{H}$, $\mathrm{CH}_{3}-\mathrm{CH}_{2}-\mathrm{CH}_{2}$ ), 2.32 (t, $J=7.2 \mathrm{~Hz}, 2 \mathrm{H}, \mathrm{CH}_{3}-\mathrm{CH}_{2}-\mathrm{CH}_{2}$ ), $6.12\left(\mathrm{~s}, 2 \mathrm{H}, \mathrm{CH}_{2}\right), 7.62$ (dt, J= 7.6 and 1.2 $\mathrm{Hz}, 2 \mathrm{H}, \mathrm{H}-2$ and H-7), 7.79 (dt, $J=7.8$ and $1.2 \mathrm{~Hz}, 2 \mathrm{H}, \mathrm{H}-3$ and H-6), 8.28 (d, $J=8.6 \mathrm{~Hz}, 2 \mathrm{H}, \mathrm{H}-4$ and H-5), 8.34 (d, $J=9.0 \mathrm{~Hz}, 2 \mathrm{H}, \mathrm{H}-1$ and H-8). ${ }^{13} \mathrm{C}$ NMR $\left(100.6 \mathrm{MHz}, \mathrm{CDCl}_{3}\right): \delta_{\mathrm{C}}=13.54\left(\mathrm{CH}_{3^{-}}\right.$ $\left.\mathrm{CH}_{2}-\mathrm{CH}_{2}\right), 18.34\left(\mathrm{CH}_{3}-\mathrm{CH}_{2}-\mathrm{CH}_{2}\right), 35.96\left(\mathrm{CH}_{3}-\mathrm{CH}_{2}-\mathrm{CH}_{2}\right), 57.31\left(\mathrm{CH}_{2}\right), 124.03(\mathrm{C}-1$ and $\mathrm{C}-8)$, 125.32 (C-8a and C-9a), 126.68 (C-2 and C-7), 129.97 (C-4 and C-5), 130.08 (C-3 and C-6), 137.53 (C-9), 148.46 (C-4a and C-4b), $173.42(\mathrm{C}=\mathrm{O})$. IR $(\mathrm{KBr} 1 \%): v=3067,2965,2934,2875,1737$, 1692, 1629, 1603, 1557, 1519, 1498, 1461, 1441, 1416, 1382, 1352, 1303, 1286, 1249, 1165, 1100, 1058, 1040, 1018, 976, 911, 862, 753, 733, $643 \mathrm{~cm}^{-1}$. HRMS (ESI) for $\mathrm{C}_{18} \mathrm{H}_{18} \mathrm{NO}_{2}\left[\mathrm{M}^{+}+\mathrm{H}\right]$ : calculated 280.13384, found: 280.13403 . 
2.6. Synthesis of (5-methoxy-3-oxo-3H-naphtho[2,1-b]pyran-1-yl)methyl butyrate, 9. To a solution of 1-chloromethyl-5-methoxy-3-oxo-3H-naphtho[2,1-b]pyran $3\left(0.100 \mathrm{~g}, 3.64 \times 10^{-4} \mathrm{~mol}\right)$ in dry DMF $(4 \mathrm{~mL})$, potassium fluoride $\left(0.063 \mathrm{~g}, 1.09 \times 10^{-3} \mathrm{~mol}\right)$ and butyric acid $\left(0.033 \mathrm{~mL}, 3.64 \times 10^{-4} \mathrm{~mol}\right)$ were added. The reaction was followed by TLC (ethyl acetate/light petroleum, 1:4), and stirred at room temperature for $42 \mathrm{~h}$. The solvent was removed by rotary evaporation under reduced pressure and the crude residue was purified by column chromatography using mixtures of ethyl acetate/light petroleum as eluent. Compound 9 was obtained as an oily solid $(0.083 \mathrm{~g}, 70 \%) . R_{\mathrm{f}}=0.22$ (ethyl acetate/light petroleum, 1:4). ${ }^{1} \mathrm{H} \mathrm{NMR}\left(400 \mathrm{MHz}, \mathrm{CDCl}_{3}\right): \delta_{\mathrm{H}}=1.01\left(\mathrm{t}, J=7.2 \mathrm{~Hz}, 3 \mathrm{H}, \mathrm{CH}_{3}-\mathrm{CH}_{2}-\right.$ $\mathrm{CH}_{2}$ ), 1.70-1.80 (m, $\left.2 \mathrm{H}, \mathrm{CH}_{3}-\mathrm{CH}_{2}-\mathrm{CH}_{2}\right), 2.48$ (t, $\left.J=7.2 \mathrm{~Hz}, 2 \mathrm{H}, \mathrm{CH}_{3}-\mathrm{CH}_{2}-\mathrm{CH}_{2}\right), 4.04$ (s, 3H, $\left.\mathrm{OCH}_{3}\right), 5.64\left(\mathrm{~d}, J=1.2 \mathrm{~Hz}, 2 \mathrm{H}, \mathrm{CH}_{2}\right), 6.70(\mathrm{t}, J=1.2 \mathrm{~Hz}, 1 \mathrm{H}, \mathrm{H}-2), 7.30(\mathrm{~s}, 1 \mathrm{H}, \mathrm{H}-6), 7.47-7.53$ (m, $2 \mathrm{H}, \mathrm{H}-8$ and H-9), 7.76-7.81 (m, $1 \mathrm{H}, \mathrm{H}-10), 7.95-8.10$ (m, $1 \mathrm{H}, \mathrm{H}-7) .{ }^{13} \mathrm{C}$ NMR (100.6 MHz, $\left.\mathrm{CDCl}_{3}\right): \delta_{\mathrm{C}}=13.62\left(\mathrm{CH}_{3}-\mathrm{CH}_{2}-\mathrm{CH}_{2}\right), 18.32\left(\mathrm{CH}_{3}-\mathrm{CH}_{2}-\mathrm{CH}_{2}\right), 35.95\left(\mathrm{CH}_{3}-\mathrm{CH}_{2}-\mathrm{CH}_{2}\right), 56.06\left(\mathrm{OCH}_{3}\right)$, $63.94\left(\mathrm{CH}_{2}\right), 110.81$ (C-6), 113.14 (C-2), 113.43 (C-4b), 123.94 (C-6b), 124.40 (C-7), 125.89 (C-8), 125.98 (C-9), 128.42 (C-10), 131.09 (C-6a), 146.65 (C-4a), 146.81 (C-5), 151.46 (C-1), 159.43 (C3), $172.65(\mathrm{C}=\mathrm{O})$. IR (KBr 1\%): $v=3426,3060$, 2964, 2878, 2836, 1733, 1627, 1599, 1559, 1513, 1460, 1425, 1346, 1322, 1264, 1147, 1121, 1085, 1005, 932, 864, 835, 780, 738, $703 \mathrm{~cm}^{-1}$. HRMS (ESI) for $\mathrm{C}_{19} \mathrm{H}_{19} \mathrm{O}_{5}\left[\mathrm{M}^{+}+\mathrm{H}\right]$ : calculated 327.12327, found: 327.12356 .

2.7. Synthesis of (8-methoxy-3-oxo-3H-naphtho[2,1-b]pyran-1-yl)methyl butyrate, 10. To a solution of 1-chloromethyl-8-methoxy-3-oxo-3H-naphtho[2,1-b]pyran $4\left(0.305 \mathrm{~g} 1.11 \times 10^{-3} \mathrm{~mol}\right)$ in dry DMF $(5 \mathrm{~mL})$, potassium fluoride $\left(0.193 \mathrm{~g}, 3.33 \times 10^{-3} \mathrm{~mol}\right)$ and butyric acid $\left(0.101 \mathrm{~mL}, 1.11 \times 10^{-3}\right.$ mol) were added. The reaction was followed by TLC (ethyl acetate/light petroleum, 1:4), and stirred at room temperature for $44 \mathrm{~h}$. The solvent was removed by rotary evaporation under reduced pressure and the crude residue was purified by column chromatography using mixtures of ethyl acetate/light petroleum as eluent. Compound $\mathbf{1 0}$ was obtained as oil $\left(0.257 \mathrm{~g}, 71 \%\right.$ ). $R_{\mathrm{f}}=0.28$ (ethyl acetate/light petroleum, 1:4). ${ }^{1} \mathrm{H}$ NMR $\left(400 \mathrm{MHz}, \mathrm{CDCl}_{3}\right): \delta_{\mathrm{H}}=0.99\left(\mathrm{t}, J=7.2 \mathrm{~Hz}, 3 \mathrm{H}, \mathrm{CH}_{3}-\mathrm{CH}_{2}-\right.$ $\left.\mathrm{CH}_{2}\right), 1.69-1.78\left(\mathrm{~m}, 2 \mathrm{H}, \mathrm{CH}_{3}-\mathrm{CH}_{2}-\mathrm{CH}_{2}\right), 2.45\left(\mathrm{t}, J=7.2 \mathrm{~Hz}, 2 \mathrm{H}, \mathrm{CH}_{3}-\mathrm{CH}_{2}-\mathrm{CH}_{2}\right), 3.90(\mathrm{~s}, 3 \mathrm{H}$, $\left.\mathrm{OCH}_{3}\right), 5.52\left(\mathrm{~d}, J=1.2 \mathrm{~Hz}, 2 \mathrm{H}, \mathrm{CH}_{2}\right), 6.58(\mathrm{t}, J=1.2 \mathrm{~Hz}, 1 \mathrm{H}, \mathrm{H}-2), 7.14(\mathrm{~d}, J=2.4 \mathrm{~Hz}, 1 \mathrm{H}, \mathrm{H}-7)$, $7.22(\mathrm{dd}, J=9.2$ and $2.8 \mathrm{~Hz}, 1 \mathrm{H}, \mathrm{H}-9), 7.31$ (d, $J=9.2 \mathrm{~Hz}, 1 \mathrm{H}, \mathrm{H}-10), 7.78$ (d, $J=8.8 \mathrm{~Hz}, 1 \mathrm{H}, \mathrm{H}-$

5), $7.87(\mathrm{~d}, J=9.6 \mathrm{~Hz}, 1 \mathrm{H}, \mathrm{H}-6) .{ }^{13} \mathrm{C} \mathrm{NMR}\left(100.6 \mathrm{MHz}, \mathrm{CDCl}_{3}\right): \delta_{\mathrm{C}}=13.54\left(\mathrm{CH}_{3}-\mathrm{CH}_{2}-\mathrm{CH}_{2}\right), 18.22$ $\left(\mathrm{CH}_{3}-\mathrm{CH}_{2}-\mathrm{CH}_{2}\right), 35.82\left(\mathrm{CH}_{3}-\mathrm{CH}_{2}-\mathrm{CH}_{2}\right), 55.22\left(\mathrm{OCH}_{3}\right), 63.78\left(\mathrm{CH}_{2}\right), 108.43(\mathrm{C}-7), 112.43(\mathrm{C}-4 \mathrm{~b})$, 112.72 (C-2), 117.89 (C-10), 119.86 (C-9), 123.50 (C-6b), 125.86 (C-6), 132.62 (C-5), 132.66 (C-1), 150.91 (C-4a), 153.14 (C-6a), 156.82 (C-8), 160.05 (C-3), 172.52 (C=O). IR (neat): v = 3425, 2965, 
2877, 2840, 1725, 1614, 1556, 1519, 1468, 1364, 1308, 1249, 1205, 1170, 1122, 1086, 1035, 999, $916,857,812,734,700,675 \mathrm{~cm}^{-1}$. HRMS (ESI) for $\mathrm{C}_{19} \mathrm{H}_{19} \mathrm{O}_{5}\left[\mathrm{M}^{+}+\mathrm{H}\right]$ : calculated 327.12327 , found: 327.12319.

2.8. Synthesis of (11-oxo-2,3,5,6,7,11-hexahydro-1H-pyrano[2,3-f]pyrido[3,2,1-ij]quinolin-9yl)methyl butyrate, 12. To a solution of 9-(chloromethyl)-2,3,6,7-tetrahydro- $1 H$-pyrano[2,3f]pyrido[3,2,1-ij]quinolin-11(5H)-one $6\left(0.042 \mathrm{mg}, 1.45 \times 10^{-4} \mathrm{~mol}\right)$ in dry DMF $(4 \mathrm{~mL})$, potassium fluoride $\left(0.025 \mathrm{~g}, 4.35 \times 10^{-4} \mathrm{~mol}\right)$ and butyric acid $\left(0.014 \mathrm{~mL}, 1.59 \times 10^{-4} \mathrm{~mol}\right)$ were added. The reaction was followed by TLC (ethyl acetate/light petroleum, 1:4), and stirred at room temperature for $14 \mathrm{~h}$. The solvent was removed by rotary evaporation under reduced pressure and the crude residue was purified by column chromatography using mixtures of ethyl acetate/light petroleum as eluent. Compound 12 was obtained as oil $(0.017 \mathrm{~g}, 34 \%) . R_{\mathrm{f}}=0.58$ (ethyl acetate/light petroleum, 1:4). ${ }^{1} \mathrm{H}$ NMR $\left(400 \mathrm{MHz}, \mathrm{CDCl}_{3}\right): \delta_{\mathrm{H}}=0.99\left(\mathrm{t}, J=7.2 \mathrm{~Hz}, 3 \mathrm{H}, \mathrm{CH}_{3}-\mathrm{CH}_{2}-\mathrm{CH}_{2}\right), 1.73$ (sext, $J=7.2$ $\left.\mathrm{Hz}, 2 \mathrm{H}, \mathrm{CH}_{3}-\mathrm{CH}_{2}-\mathrm{CH}_{2}\right), 1.91-2.03(\mathrm{~m}, 4 \mathrm{H}, \mathrm{H}-2$ and $\mathrm{H}-6), 2.42$ (t, J = 7.2 Hz, $2 \mathrm{H}, \mathrm{CH}_{3}-\mathrm{CH}_{2}-\mathrm{CH}_{2}$ ), $2.76(\mathrm{t}, J=6.4 \mathrm{~Hz}, 2 \mathrm{H}, \mathrm{H}-7), 2.89$ (t, $J=6.4 \mathrm{~Hz}, 2 \mathrm{H}, \mathrm{H}-1), 3.18-3.33$ (m, $4 \mathrm{H}, \mathrm{H}-3$ and H-5), 5.20 (s, $\left.2 \mathrm{H}, \mathrm{CH}_{2}\right), 6.10$ (s, $\left.1 \mathrm{H}, \mathrm{H}-10\right), 6.88$ (s, $\left.1 \mathrm{H}, \mathrm{H}-8\right) .{ }^{13} \mathrm{C} \mathrm{NMR}\left(100.6 \mathrm{MHz}, \mathrm{CDCl}_{3}\right): \delta_{\mathrm{C}}=13.66$ $\left(\mathrm{CH}_{3}-\mathrm{CH}_{2}-\mathrm{CH}_{2}\right), 18.37\left(\mathrm{CH}_{3}-\mathrm{CH}_{2}-\mathrm{CH}_{2}\right), 20.39$ (C-2), 20.55 (C-1), 21.45 (C-6), 27.67 (C-7), 36.00 $\left(\mathrm{CH}_{3}-\mathrm{CH}_{2}-\mathrm{CH}_{2}\right), 49.48(\mathrm{C}-3), 49.88(\mathrm{C}-5), 61.17\left(\mathrm{CH}_{2}\right), 105.49$ (C-10), $105.80(\mathrm{C}-8 \mathrm{a}), 106.96$ (C12b), 118.20 (C-7a), 120.40 (C-8), 145.95 (C-7b), 149.68 (C-9), 151.17 (C-12a), 162.26 (C-11), $172.93(\mathrm{C}=\mathrm{O})$. IR (neat): $v=2958,2933,2873,2856,1720,1605,1558,1522,1437,1382,1343$, $1312,1203,1174,1124,1075,1019,884,851,753,738,701 \mathrm{~cm}^{-1}$. HRMS (ESI) for $\mathrm{C}_{20} \mathrm{H}_{24} \mathrm{NO}_{4}$ $\left[\mathrm{M}^{+}+\mathrm{H}\right]$ : calculated 342.17062, found: 342.17031 .

2.9. Synthesis of (5-methoxy-3-thioxo-3H-naphtho[2,1-b]pyran-1-yl)methyl butyrate, 13. Lawesson's reagent $\left(0.268 \mathrm{~g}, 6.62 \times 10^{-4} \mathrm{~mol}\right)$ was added to a solution of (5-methoxy-3-oxo-3H-naphtho[2,1b]pyran-1-yl)methyl butyrate $9\left(0.072 \mathrm{~g}, 2.20 \times 10^{-4} \mathrm{~mol}\right)$ in toluene $(8 \mathrm{~mL})$. The reaction mixture was refluxed for $9 \mathrm{~h}$ and the process was followed by TLC (ethyl acetate/light petroleum, 1:4). The solvent was removed by rotary evaporation under reduced pressure and the crude residue was purified by column chromatography using dichlorometane/light petroleum mixtures of increasing polarity as eluent. Compound 13 was obtained as oil $(0.004 \mathrm{~g}, 5 \%) . R_{\mathrm{f}}=0.71$ (ethyl acetate/light petroleum, 1:4). ${ }^{1} \mathrm{H} \mathrm{NMR}\left(400 \mathrm{MHz}, \mathrm{CDCl}_{3}\right): \delta_{\mathrm{H}}=1.02\left(\mathrm{t}, J=7.2 \mathrm{~Hz}, 3 \mathrm{H}, \mathrm{CH}_{3}-\mathrm{CH}_{2}-\mathrm{CH}_{2}\right), 1.70$ $1.81\left(\mathrm{~m}, 2 \mathrm{H}, \mathrm{CH}_{3}-\mathrm{CH}_{2}-\mathrm{CH}_{2}\right), 2.50\left(\mathrm{t}, J=7.2 \mathrm{~Hz}, 2 \mathrm{H}, \mathrm{CH}_{3}-\mathrm{CH}_{2}-\mathrm{CH}_{2}\right), 4.10\left(\mathrm{~s}, 3 \mathrm{H}, \mathrm{OCH}_{3}\right), 5.66$ (d, $J=1.2 \mathrm{~Hz}, 2 \mathrm{H}, \mathrm{CH}_{2}$ ), 7.39 (s, $\left.1 \mathrm{H}, \mathrm{H}-6\right), 7.52-7.62$ (m, $3 \mathrm{H}, \mathrm{H}-2, \mathrm{H}-8$ and H-9), 7.81-7.86 (s, $1 \mathrm{H}$, 
$\mathrm{H}-7), 8.03-8.10(\mathrm{~m}, 1 \mathrm{H}, \mathrm{H}-10) .{ }^{13} \mathrm{C} \mathrm{NMR}\left(100.6 \mathrm{MHz}, \mathrm{CDCl}_{3}\right): \delta_{\mathrm{C}}=13.66\left(\mathrm{CH}_{3}-\mathrm{CH}_{2}-\mathrm{CH}_{2}\right), 18.39$ $\left(\mathrm{CH}_{3}-\mathrm{CH}_{2}-\mathrm{CH}_{2}\right), 35.99\left(\mathrm{CH}_{3}-\mathrm{CH}_{2}-\mathrm{CH}_{2}\right), 56.24\left(\mathrm{OCH}_{3}\right), 63.76\left(\mathrm{CH}_{2}\right), 111.27(\mathrm{C}-6), 116.07(\mathrm{C}-4 \mathrm{~b})$, 123.76 (C-6b), 125.02 (C-10), 126.24 (C-9), 126.73 (C-8), 127.80 (C-2), 128.57 (C-7), 131.63 (C6a), 142.49 (C-1), 146.33 (C-5), 150.29 (C-4a), 172.78 (C=O), 194.49 (C-3). IR (neat): v = 2964, 2932, 2875, 1738, 1623, 1593, 1545, 1513, 1460, 1425, 1323, 1297, 1256, 1233, 1218, 1164, 1125, 1104, 1004, 913, 866, 835, 778, $739 \mathrm{~cm}^{-1}$. HRMS (ESI): calculated for $\mathrm{C}_{19} \mathrm{H}_{19} \mathrm{O}_{4} \mathrm{~S}\left[\mathrm{M}^{+}+\mathrm{H}\right]$ : 343.10047, found: 343.10079 .

2.10. Synthesis of (8-methoxy-3-thioxo-3H-naphtho[2,1-b]pyran-1-yl)methyl butyrate, 14. Lawesson's reagent $\left(0.383 \mathrm{~g}, 9.47 \times 10^{-4} \mathrm{~mol}\right)$ was added to a solution of (8-methoxy-3-oxo-3Hnaphtho[2,1-b]pyran-1-yl)methyl butyrate $10\left(0.103 \mathrm{~g}, 3.16 \times 10^{-4} \mathrm{~mol}\right)$ in toluene $(8 \mathrm{~mL})$. The reaction mixture was refluxed for $23 \mathrm{~h}$ and followed by TLC (ethyl acetate/light petroleum, 1:4). The solvent was removed by rotary evaporation under reduced pressure and the crude residue was purified by column chromatography using ethyl acetate/light petroleum mixtures of increasing polarity as eluent. Compound 14 was obtained as an orange solid (0.087 g, 80\%). M.p. 144.9-146.5 ${ }^{\circ} \mathrm{C} . R_{\mathrm{f}}=0.64$ (ethyl acetate/light petroleum, 1:4). ${ }^{1} \mathrm{H}$ NMR $\left(400 \mathrm{MHz}, \mathrm{CDCl}_{3}\right): \delta_{\mathrm{H}}=1.02(\mathrm{t}, J=7.2$ $\left.\mathrm{Hz}, 3 \mathrm{H}, \mathrm{CH}_{3}-\mathrm{CH}_{2}-\mathrm{CH}_{2}\right), 1.70-1.80\left(\mathrm{~m}, 2 \mathrm{H}, \mathrm{CH}_{3}-\mathrm{CH}_{2}-\mathrm{CH}_{2}\right), 2.49$ (t, J = 7.2 Hz, $2 \mathrm{H}, \mathrm{CH}_{3}-\mathrm{CH}_{2}-$ $\mathrm{CH}_{2}$ ), 3.96 (s, $3 \mathrm{H}, \mathrm{OCH}_{3}$ ), 5.57 (d, $J=0.8 \mathrm{~Hz}, 2 \mathrm{H}, \mathrm{CH}_{2}$ ), 7.24 (d, J=2.8 Hz, $\left.1 \mathrm{H}, \mathrm{H}-7\right), 7.29-7.35$ (m, $1 \mathrm{H}, \mathrm{H}-9), 7.51$ (s, $1 \mathrm{H}, \mathrm{H}-2), 7.55$ (d, J = 9.2 Hz, $1 \mathrm{H}, \mathrm{H}-10), 7.91$ (d, J = 9.2 Hz, $1 \mathrm{H}, \mathrm{H}-5$ ), 8.02 $(\mathrm{d}, J=9.6 \mathrm{~Hz}, 1 \mathrm{H}, \mathrm{H}-6) .{ }^{13} \mathrm{C} \mathrm{NMR}\left(100.6 \mathrm{MHz}, \mathrm{CDCl}_{3}\right): \delta_{\mathrm{C}}=13.63\left(\mathrm{CH}_{3}-\mathrm{CH}_{2}-\mathrm{CH}_{2}\right), 18.34\left(\mathrm{CH}_{3^{-}}\right.$ $\left.\mathrm{CH}_{2}-\mathrm{CH}_{2}\right), 35.93\left(\mathrm{CH}_{3}-\mathrm{CH}_{2}-\mathrm{CH}_{2}\right), 55.43\left(\mathrm{OCH}_{3}\right), 63.66\left(\mathrm{CH}_{2}\right), 108.83(\mathrm{C}-7), 115.09(\mathrm{C}-4 \mathrm{~b}), 117.77$ (C-10), 120.24 (C-9), 123.26 (C-6b), 126.60 (C-6), 127.22 (C-2), 133.23 (C-6a), 133.39 (C-5), 142.29 (C-1), 156.87 (C-4a), $157.56(\mathrm{C}-8), 172.71(\mathrm{C}=\mathrm{O}), 195.02(\mathrm{C}-3) . \mathrm{IR}(\mathrm{KBr} 1 \%): v=3061$, 2961, 2877, 1903, 1743, 1609, 1542, 1524, 1468, 1454, 1426, 1367, 1292, 1266, 1208, 1163, 1139, 1097, 1035, 978, 936, 908, 858, 810, $736 \mathrm{~cm}^{-1}$. HRMS (ESI) for $\mathrm{C}_{19} \mathrm{H}_{19} \mathrm{O}_{4} \mathrm{~S}\left[\mathrm{M}^{+}+\mathrm{H}\right]$ : calculated 343.10047, found: 343.10096 .

2.11. Synthesis of (9-methoxy-3-thioxo-3H-naphtho[2,1-b]pyran-1-yl)methyl butyrate, 15. Lawesson's reagent $\left(0.166 \mathrm{~g}, 4.11 \times 10^{-4} \mathrm{~mol}\right)$ was added to a solution of (9-methoxy-3-oxo-3Hbenzo[f]benzopyran-1-yl)methyl butyrate, $11\left(0.067 \mathrm{~g}, 2.05 \times 10^{-4} \mathrm{~mol}\right)$ in toluene $(5 \mathrm{~mL})$. The reaction mixture was heated at reflux for $48 \mathrm{~h}$ and the process was followed by TLC (ethyl acetate/ light petroleum, 1:4). The solvent was removed by rotary evaporation under reduced pressure and the crude residue was purified by column chromatography using ethyl acetate/light petroleum, with 
mixtures of increasing polarity as eluent. Compound 15 was obtained as an orange solid (0.049 g, 70\%). Mp 132.5-134.7 ${ }^{\circ} \mathrm{C} . R_{\mathrm{f}}=0.86$ (ethyl acetate/light petroleum, 1:4). ${ }^{1} \mathrm{H}$ NMR (400 MHz, $\left.\mathrm{CDCl}_{3}\right): \delta_{\mathrm{H}}=1.01\left(\mathrm{t}, J=7.2 \mathrm{~Hz}, 3 \mathrm{H}, \mathrm{CH}_{3}-\mathrm{CH}_{2}-\mathrm{CH}_{2}\right.$ ), 1.75 (sext, $J=7.2 \mathrm{~Hz}, 2 \mathrm{H}, \mathrm{CH}_{3}-\mathrm{CH}_{2}-\mathrm{CH}_{2}$ ), $2.49\left(\mathrm{t}, J=7.6 \mathrm{~Hz}, 2 \mathrm{H}, \mathrm{CH}_{3}-\mathrm{CH}_{2}-\mathrm{CH}_{2}\right), 3.97\left(\mathrm{~s}, 3 \mathrm{H}, \mathrm{OCH}_{3}\right), 5.60$ (s, $\left.2 \mathrm{H}, \mathrm{CH}_{2}\right), 7.24$ (dd, $J=8.8$ and $2.4 \mathrm{~Hz}, 1 \mathrm{H}, \mathrm{H}-8), 7.43$ (d, $J=9.2 \mathrm{~Hz}, 1 \mathrm{H}, \mathrm{H}-5), 7.48$ (d, $J=2.4 \mathrm{~Hz}, 1 \mathrm{H}, \mathrm{H}-10), 7.49$ (s, $1 \mathrm{H}$, $\mathrm{H}-2), 7.83(\mathrm{~d}, J=8.8 \mathrm{~Hz}, 1 \mathrm{H}, \mathrm{H}-7), 7.93$ (d, $J=8.8 \mathrm{~Hz}, 1 \mathrm{H}, \mathrm{H}-6) .{ }^{13} \mathrm{C}$ NMR $\left(100.6 \mathrm{MHz}, \mathrm{CDCl}_{3}\right)$ : $\delta_{\mathrm{C}}=13.63\left(\mathrm{CH}_{3}-\mathrm{CH}_{2}-\mathrm{CH}_{2}\right), 18.28\left(\mathrm{CH}_{3}-\mathrm{CH}_{2}-\mathrm{CH}_{2}\right), 35.92\left(\mathrm{CH}_{3}-\mathrm{CH}_{2}-\mathrm{CH}_{2}\right), 55.47\left(\mathrm{OCH}_{3}\right), 63.69$ $\left(\mathrm{CH}_{2}\right), 106.25$ (C-10), 114.30 (C-4b), 114.90 (C-5), 117.22 (C-8), 126.56 (C-6a), 126.97 (C-2), 130.29 (C-6b), 131.38 (C-7), 134.25 (C-6), 142.39 (C-1), 158.69 (C-4a), 159.86 (C-9), 172.76 $(\mathrm{C}=\mathrm{O}), 195.27$ (C-3). IR (KBr 1\%): $v=3074,2964,2935,2875,2836,1742,1623,1606,1592$, 1538, 1485, 1461, 1443, 1431, 1364, 1296, 1229, 1216, 1163, 1139, 1096, 1056, 1025, 1008, 982, $917,870,838,803,706 \mathrm{~cm}^{-1}$. HRMS (ESI) for $\mathrm{C}_{19} \mathrm{H}_{19} \mathrm{O}_{4} \mathrm{~S}\left[\mathrm{M}^{+}+\mathrm{H}\right]$ : calculated 343.10047, found: 343.10074.

\subsection{Photolysis general}

A $1 \times 10^{-4} \mathrm{M}$ methanol or acetonitrile/HEPES (80:20) solution of compounds 7-15 (5 mL) were placed in a quartz tube and irradiated in a Rayonet RPR-100 reactor at the desired wavelength. The lamps used for irradiation were of 254, 300, 350 and $419 \pm 10 \mathrm{~nm}$. HEPES buffer solution was prepared in distilled water with HEPES (4-(2-hydroxyethyl)-1-piperazine ethanesulfonic acid) (10 $\mathrm{mM})$, sodium chloride $(120 \mathrm{mM})$, potassium chloride $(3 \mathrm{mM})$, calcium chloride $(1 \mathrm{mM})$ and magnesium chloride $(1 \mathrm{mM})$ and $\mathrm{pH}$ adjusted to 7.2 with aqueous $1 \mathrm{M}$ sodium hydroxide solution.

Aliquots of $100 \mu \mathrm{L}$ were taken at regular intervals and analysed by RP-HPLC using a Licrospher 100 RP18 $(5 \mu \mathrm{m})$ column in a JASCO HPLC system composed by a PU-2080 pump and a UV-2070 detector with ChromNav software. The eluent was acetonitrile/water, 75:25 at a flow rate of 0.8 $\mathrm{mL} / \mathrm{min}$, previously filtered through a Millipore, type $\mathrm{HN} 0.45 \mu \mathrm{m}$ filter and degassed by ultra-sound for $30 \mathrm{~min}$. The chromatograms were traced by detecting UV absorption at the wavelength of maximum absorption for each compound (retention time: $7,5.2 ; \mathbf{8}, 8.7 ; \mathbf{9}, 6.1 ; \mathbf{1 0}, 6.6 ; \mathbf{1 1}, 6.5 ; \mathbf{1 2}$, $8.4 ; 13,9.4 ; 14,10.4 ; 15,8.5 \mathrm{~min})$.

\subsection{Time-resolved fluorescence measurements}

Time-resolved fluorescence measurements with one photon excitation made use of the timecorrelated single-photon counting (TCSPC) technique and were performed using a HORIBA Scientific DeltaFlex, equipped with a DeltaDiode laser excitation source emitting at $378 \mathrm{~nm}$ and a 
PPD-850 detector. Analysis of each dataset was performed using DAS6 software and the decays were reconvoluted with the instrumental response and fitted to the sum of exponentials (equation 1).

$$
I(t)=\sum_{i=1}^{n} \alpha_{i} \exp ^{-t} / \tau_{i}
$$

Where $\alpha_{\mathrm{i}}$ are the normalised pre-exponential factors and the average lifetime was obtained from

$$
\tau_{\text {ave }}=\Sigma \alpha_{i} \tau_{i}
$$

The goodness of fit was judged in terms of a chi-squared value and weighted residuals.

Two-photon excitation (TPE) TCSPC measurements were performed using Ti:sapphire $80 \mathrm{MHz}$ pulsed laser (Chameleon ${ }^{\circledR}$, Coherent, UK) equipped with a pulse selector (A.P.E. PulseSelect) picking 1:20 pulses generating an emission train at 4MHz. The fluorescence was recorded using a Horiba Scientific FluoroCube synchronized to the pulse train by the driving unit of the pulse selector. The emission signal was recorded using a blue green copper sulphate bandpass filter. The detector used was a Horiba Scientific TBX-850 and data were analysed in a similar manner to the one photon excitation data using DAS6 software. The TPE cross-sections, expressed in Göppert-Meyer units (GM) with $1 \mathrm{GM}=10^{-50} \mathrm{~cm}^{4} \mathrm{~s}_{\text {photon }}{ }^{-1}$ [27], were calculated relative to a reference fluorophore in a manner that we have previously reported [28]. Briefly to obtain the raw data the Ti:sapphire laser was tuned from $690 \mathrm{~nm}$ to $850 \mathrm{~nm}$ and the counts-per-second on the Horiba TBX detector recorded.

\section{Results and discussion}

\subsection{Synthesis of butyric acid conjugates 7-15}

Butyric acid was chosen as a model carboxylic acid drug to study the behaviour towards irradiation of the corresponding cages with several light sensitive $o$-nitrobenzyl or heterocyclic benzyl type esters 7-12. Their synthesis started with the preparation of the 2-nitrobenzyl ester cage 7, by a $N, N^{\prime}-$ dicyclohexylcarbodiimide (DCC)/1-hydroxybenzotriazole (HOBt) mediated coupling of butyric acid with (2-nitrophenyl)methanol $\mathbf{1}$ in DMF at room temperature. For the assembly of the heterocyclic benzyl type ester cages 8-12, several bromo- or chloromethylated heteroaromatics based on acridine 2, naphtho[2,1-b]pyran 3-5 and a 3H-benzopyran fused julolidine $\mathbf{6}$ were reacted with the model acid in the presence of potassium fluoride in DMF at room temperature (Scheme 1, Table 1). Fused pyrans 3-6 resulted from a Pechmann condensation between ethyl 4-chloro-3-oxobutanoate and 3methoxy-2-naphthol, 6-methoxy-2-naphthol, 7-methoxy-2-naphthol [25] and 1,2,3,5,6,7- 
hexahydropyrido[3,2,1-ij]quinolin-8-ol (trivially named 8-hydroxyjulolidine) [26], respectively. With the purpose of replacing the carbonyl by a thiocarbonyl group at the naphtho[2,1-b]pyran conjugates 9-11, these compounds were reacted with Lawesson's reagent in toluene, under reflux conditions [29], affording the corresponding thionaphthopyran conjugates 13-15.

\section{< Scheme 1> \\ < Table 1>}

All compounds were fully characterised by high resolution mass spectrometry, IR, ${ }^{1} \mathrm{H}$ and ${ }^{13} \mathrm{C}$ NMR spectroscopy. The IR spectra of compounds 7-15 displayed stretching vibration bands of the ester carbonyl group from 1711 to $1743 \mathrm{~cm}^{-1}$. ${ }^{1} \mathrm{H}$ NMR spectra showed signals of butyric acid, the methyl $(\delta$ 0.89-1.02 $\mathrm{ppm})$ and two methylenes ( $\delta 1.58-1.81$ and $2.32-2.50 \mathrm{ppm})$. The heterocycle methylene group, adjacent to the ester link, was visible for all compounds ( $\delta$ 5.20-6.12 ppm). The newly formed ester linkages were confirmed by ${ }^{13} \mathrm{C}$ NMR spectra signals of the carbonyl group, at about $\delta 172.52$ $173.42 \mathrm{ppm}$. The thiocarbonyl group in compounds 13-15 affected the chemical shift of the pyran proton $\mathrm{H}-2$, which appeared downfield in the range $\delta 7.51-7.59 \mathrm{ppm}$, while in the precursor compounds 9-11, with a carbonyl group, it occurred at $\delta 6.58-6.70 \mathrm{ppm}$. The presence of the new $\mathrm{C}=\mathrm{S}$ bond (C-3) at the heterocyclic ring was also confirmed by ${ }^{13} \mathrm{C}$ NMR spectra signals at $\delta$ 194.49$195.27 \mathrm{ppm}$, when compared to the carbonyl group, which occurred at $\delta 159.43-160.31 \mathrm{ppm}$. The chemical shift of the pyran carbon C-2 was also influenced by the carbon-sulphur double bond, being in the range $\delta 126.97-127.80 \mathrm{ppm}$ for compounds $\mathbf{1 3 - 1 5}$, and $\delta 112.72$ or $113.14 \mathrm{ppm}$ for compounds 9-11.

\subsection{Evaluation of the photophysical properties of butyric acid conjugates 7-15}

Fundamental UV/visible photophysical characterisation was carried out to obtain the parameters required for monitoring the photolytic process. The absorption and emission spectra of degassed $10^{-5}$ M solutions in absolute ethanol, a methanol/HEPES and acetonitrile/HEPES buffer (80:20) solutions of ester conjugates 7-15 were measured and the corresponding data, absorption and emission maxima, molar absorption coefficients and relative fluorescence quantum yields are reported in Table 1. Relative fluorescence quantum yields were calculated using 9,10-diphenylanthracene in ethanol $\left(\Phi_{\mathrm{F}} 0.95\right)[30]$ or a $0.05 \mathrm{M}$ solution of quinine in sulphuric acid ( $\left.\Phi_{\mathrm{F}} 0.546\right)[31]$ as standards. For the $\Phi_{\mathrm{F}}$ determination, the fluorescence standard was excited at the wavelengths of maximum 
absorption found for each compound to be tested and in all fluorimetric measurements the absorbance of the solution did not exceed 0.1 .

Regarding the maximum absorption wavelengths $\left(\lambda_{\mathrm{abs}}\right)$ of conjugates $\mathbf{7 - 1 5}$, in all the tested solvents, it was found that the nitrobenzyl conjugate 7 displayed the lowest values (at about $260 \mathrm{~nm}$ ), in comparison with the polycyclic compounds 8-12 (344-394 nm), whereas the thiocarbonyl conjugates 13-15, displayed even larger bathochromic shifts (404-431 nm). For naphtho[2,1-b]pyrans 9-11, which differ in the relative position of the electron donor methoxy group, the $\lambda_{\text {abs }}$ was longer for substitution in position 8 (compound 10), with a bathochromic shift between 15 to $23 \mathrm{~nm}$, when compared with substitution in positions 5 and 9 (compounds 9 and 11, respectively). Nevertheless, the largest shift was observed for the $3 H$-benzopyran fused julolidine 12, with maximum absorption wavelengths in the range 394-399 nm, in the various solvents. Furthermore, the replacement of the carbonyl group in the pyran ring (compounds 9-11) by a thiocarbonyl (compounds 13-15) also resulted in a shift in the $\lambda_{\mathrm{abs}}$, by tuning absorption to values in the visible region ( $\left.\lambda_{\text {abs }} 404-431 \mathrm{~nm}\right)$.

Concerning the fluorescence spectra, in all the tested solvents, it was observed that emission maxima $\left(\lambda_{\mathrm{em}}\right)$ of conjugates 8-15 occurred in the range $(410-497 \mathrm{~nm})$, being the $3 H$-benzopyran fused julolidine 12 associated to the longer wavelengths, and the naphtho[2,1-b]pyran $\mathbf{1 0}$ the most emissive ( $\Phi_{\mathrm{F}} 0.45$ to 0.53 ). The nitrobenzyl conjugate 7 displayed the lowest values ( $\lambda_{\mathrm{em}}$ at about $300 \mathrm{~nm}$ ), as was expected due to its structure. The emission of thiocarbonyl conjugates 13-15 was hypsochromically shifted with lower fluorescent quantum yields, in comparison with the corresponding carbonyl precursors.

\subsection{Photolysis studies of butyric acid conjugates 7-15}

The present work intended to profit from our gathered knowledge in the application of different (hetero)aromatic benzyl type systems as photoremovable protecting groups for a variety of relevant biomolecules. In particular, our previous findings in the photolytic release of butyric acid from different naphthoxazole, naphthopyran and oxazole fused pyran cages, revealed that these heterocyclic systems were promising mainly at short wavelengths. In order to develop a system that allows the delivery of butyric acid at longer wavelengths, heterocyclic compounds that showed interesting results with other biomolecules, as previously reported [16,18,24], as well as new naphthopyrans methoxylated at different positions of the polycyclic ring, and the corresponding thionated derivatives were used in the caging of butyric acid. Photolytic studies of all the ester cages 7-15 were carried out in a Rayonet RPR-100 reactor at 254, 300, 350 and $419 \mathrm{~nm}$ in mixtures of methanol or acetonitrile with aqueous HEPES buffer in 80:20 solutions, with collection of kinetic 
data. The course of the photocleavage reaction was followed by reverse phase HPLC with UV detection. The plots of peak area $(A)$ of the starting material versus irradiation time were obtained for each compound, at the considered wavelengths. Peak areas were determined by HPLC, which revealed a gradual decrease with time, and were the average of three runs. The determined irradiation time represents the time necessary for the consumption of the starting materials until less than $5 \%$ of the initial area was detected (Table 2). For each compound and based on HPLC data, the plot of $\ln A$ versus irradiation time showed a linear correlation for the disappearance of the starting material, which suggested a first order reaction, obtained by the linear least squares methodology for a straight line. The photochemical quantum yields $\left(\Phi_{\text {Phot }}\right)$ were calculated based on half-lives $\left(t_{1 / 2}\right)$, molar absorptivities $(\varepsilon)$ and the incident photon flux $\left(I_{0}\right)$, which was determined by potassium ferrioxalate actinometry [30].

The results at various wavelengths of irradiation revealed the significant influence of the photoactive unit structure in the irradiation time $\left(t_{\text {irr }}\right)$ necessary to release butyric acid (Table 2$)$. Although a study at short wavelengths (254 and $300 \mathrm{~nm}$ ) was carried out for comprehensiveness and the results are included in Table 2, the main focus of the present work was the performance at 350 and $419 \mathrm{~nm}$. At these wavelengths, considering the nitrogen heterocyclic system, namely acridine cage $\mathbf{8}$, it was found that the release occurred with very short $t_{\text {irr }}$ in methanol/HEPES when compared to acetonitrile/HEPES, which suggested the influence of the organic solvent character in this photoreaction. For the remaining oxygen heterocyclic conjugates, naphtho[2,1-b]pyrans 9-11, irradiation times were comparable in both solvent systems, with compound $\mathbf{9}$ cleaving faster at 350 $\mathrm{nm}$ and compound $\mathbf{1 1}$ at $419 \mathrm{~nm}$, which can be related to the position of the methoxy substituent. However, the best results were obtained with the $3 H$-benzopyran fused julolidine 12, at both wavelengths and solvent systems, as butyric acid was completely released within 4 to 6 min of irradiation. The bathochromic shift in the maximum absorption wavelengths related to the presence of the thiocarbonyl group in cages 13-15 lead to significantly shorter irradiation times at $419 \mathrm{~nm}$ due to an increase in the efficiency of the photolysis, in comparison to the carbonyl precursors 9-11, as expected.

<Table 2>

Additionally to monitoring the photolysis process by HPLC/UV detection, the release of butyric acid was also followed by ${ }^{1} \mathrm{H}$ NMR in an acetonitrile- $d_{3} / \mathrm{D}_{2} \mathrm{O}(80: 20)$ solution. Upon irradiation at 419 
$\mathrm{nm}$ of a solution of $3 \mathrm{H}$-benzopyran fused julolidine cage 12, the signal due to the benzylic-type $\mathrm{CH}_{2}$ at position 9 of the heterocyclic ring, visible at about $\delta 5.17 \mathrm{ppm}$ gradually decreased with time. The same observation occurred with the signals related to the butyric acid in the conjugated form at about $\delta 2.45,1.65$ and $0.95 \mathrm{ppm}$, giving rise to a close set of signals corresponding to butyric acid in its free form at about $\delta 2.10,1.55$ and $0.90 \mathrm{ppm}$, respectively (Figure 1). NMR monitoring was carried out with a $1.17 \times 10^{-2} \mathrm{M}$ solution, which led to an expected increase in the photolysis time for the complete release of the molecule, when compared to the irradiation times in Table 2 obtained with dilute solutions.

\subsection{Time-resolved studies of butyric acid conjugates 8-15}

To help elucidate the photophysical processes occurring in these compounds time-resolved fluorescence measurements were performed on samples that were freshly prepared (after an initial check of the absorption and emission steady state spectra). To facilitate future (accessible using 2photon excitation) studies, an excitation wavelength of $378 \mathrm{~nm}$ for the compounds in acetonitrile/HEPES buffer (80:20) solution was chosen. This wavelength is between the two longer ones employed in the photoreactor and provides an excitation into the main absorption bands for each of the compounds. Measurements were made close to the peak emission and in every case the decay was found to require the sum of 3 exponential components (apart from 2 exponentials for 8). This is indicative of the presence of different excited state species and the outcome of these measurements is presented in Table 3. The behaviour seen is in keeping with our previous observations $[19,20,26]$ and the longer-lived emission can relate to unquenched fluorophore. The shorter-lived decays are attributed to charge transfer states, caused by the presence of the conjugate upon excitation, with the possibility of the formation of an ion pair that can either recombine or progress to a cleaved pair. Thus, the contribution of each of the fluorescing components gives an insight into the species present.

Here we aim to ascertain if the fluorescence lifetime can help provide complementary information to that of the photolysis study and assume that the amount of photocleavage during the measurement is not too significant. From Table 3 it can be noted that the compound giving the highest photochemical yield (12) also exhibits the shortest average lifetime, showing that overall the non-radiative processes, ie charge transfer, can be higher in this compound. On the other hand, compound $\mathbf{8}$, considering the $350 \mathrm{~nm}$ values in Table 2, has one of the lowest photochemical yields and exhibits the longest-lived average lifetime. It is a moot point in this case whether the quantity of the longestlived component relates to cleaved photoproduct, as we have previously shown using decay associated spectra and a kinetic measurement [26], or can reflect on the strength of coupling to the 
conjugate. This will be the basis of further work, although the fact that $\mathbf{1 2}$ exhibits a high photochemical yield, while displaying the shortest average lifetime may indicate the latter. Here the photophysics will be used to assess the effect of treatment of Lawesson's reagent (ie comparison of 9,10,11 with 13,14,15 respectively). Considering the data in Table 3, it does not appear that treatment dramatically affects the lifetime values, minor changes are observed and their trend between compounds similar before and after treatment with Lawesson's reagent. However there are some differences in the contribution of the lifetimes to the overall emission. It would appear that the use of Lawesson's reagent has generally had the desired effect of enabling longer wavelength irradiation to be employed without significantly altering the photophysics of these compounds.

Having established that longer wavelength excitation could be employed, and since this fits within the wavelength range of Ti:sapphire lasers making use of two-photon excitation, a study was performed to ascertain the compounds two-photon absorption cross-section. Initially to investigate the extent of two-photon excitation the relation between the fluorescence intensity and the excitation power was plotted. Figure $2 \mathrm{a}$ shows an example of the intensity squared dependence of the fluorescence signal form the measurement of compound 11. The slope of the curve lies close to 2 , which is indicative of a two-photon excitation process. Measurements for the other compounds yielded similar dependencies between the fluorescence intensity and the excitation power. An example of a decay measurement (this time compound 10) obtained using $750 \mathrm{~nm}$ excitation is shown in Figure $2 \mathrm{~b}$. The lifetime values recovered $\left(\tau_{1}=378 \pm 56 \mathrm{ps}, \alpha_{1}=0.29 ; \tau_{2}=4948 \pm 174 \mathrm{ps}\right.$, $\alpha_{2}=0.44 ; \tau_{3}=9635 \pm 84 \mathrm{ps}, \alpha_{3}=0.27$ ) are in keeping with those obtained from the one-photon excitation measurement. It should be noted that the pulses appearing in the instrumental response arise from suppression of the fundamental $80 \mathrm{MHz}$ pulse train by the pulse picker. Their presence has been reduced to $\sim 1 \%$ of the principal pulse and appear accounted for in the fitting process, as judged by the weighted residuals.

\section{<Figure 2>}

To further characterise their suitability the two-photon absorption cross-section were obtained, and these are given in Figure 3. The probability for two-photon-excitation (TPE) depends on both spatial and temporal overlap of the incident photons. TPE spectra will usually differ from one-photon spectra as transitions that are forbidden in one case may be allowed in the other [33]. The cross sections for TPE are usually small and very high photon flux is needed for excitation. The measured GM for different fluorophores can range from 1 to 1000 GM [34]. From Figure 3, peak values of 
about $8 \mathrm{GM}$ for compounds $\mathbf{1 0}$ and $\mathbf{1 1}$ are obtained. Weaker cross-sections are obtained for the compounds treated with Lawesson's reagent, with TPE characteristics reaching up to 2, although their spectral response extends to longer wavelengths. However, it should also be noted that because of the uncertainty reported for the reference values [35] and subsequent error progression in the calculation these values should be treated as good estimates. Nevertheless, TPE cross-sections falling in the range of 1 to $10 \mathrm{GM}$ are in good agreement with commonly used dyes [34, 36], although weaker than those exhibited by engineered fluorescent proteins [33]. Thus it is apparent that these compounds are indeed suitable for two-photon excitation.

\section{<Figure 3>}

\section{Conclusions}

Acridine, naphtho[2,1-b]pyran, 3H-benzopyran fused julolidine and thioxo-naphtho[2,1- $b]$ pyran ester cages of butyric acid were studied for the controlled delivery of the active molecule by photolysis at selected wavelengths $(254,300,350$ and $419 \mathrm{~nm})$, in comparison with the well-known $o$-nitrobenzyl photolabile group. Irradiation was carried out in a Rayonet RPR-100 photochemical reactor in mixtures of organic solvents (methanol or acetonitrile) with aqueous HEPES buffer and monitored by HPLC-UV and ${ }^{1} \mathrm{H}$ NMR. Overall, the obtained results, combined with time-resolved fluorescence data, showed that for naphtho[2,1-b]pyrans the attachment position of the methoxy substituent as well as the presence of the thiocarbonyl group influenced the behaviour towards light of the corresponding cage. Furthermore, the release of butyric acid was faster in the case of using (acridin-9-yl)methyl, and [11-oxo-2,3,5,6,7,11-hexahydro-1H-pyrano[2,3-f]pyrido[3,2,1-ij]quinolin9-yl]methyl (3H-benzopyran fused julolidine) groups as phototriggers. The suitability to be addressed using two-photon excitation was also demonstrated with promising TPE cross-sections.

\section{Acknowledgements}

Thanks are due to the Fundação para a Ciência e Tecnologia (FCT, Portugal) for financial support to the NMR portuguese network (PTNMR, Bruker Avance III 400-Univ. Minho), FCT and FEDER (European Fund for Regional Development)-COMPETE-QREN-EU for financial support to the research centre CQ/UM [PEst-C/QUI/UI0686/2013 (FCOMP-01-0124-FEDER-037302)] and a PhD grant to A.M.P. (SFRH/BD/61459/2009) is also acknowledged. 


\section{References}

[1] A.L. Simplício, J.M. Clancy, J.F. Gilmer, Prodrugs for amines, Molecules 13 (2008) 519-547.

[2] G.A. Ellis, N.A. McGrath, M.J. Palte, R.T. Raines, Ribonuclease-activated cancer prodrug, ACS Med. Chem. Lett. 3 (2012) 268-272.

[3] P.K. Vemula, G.A. Cruikshank, J.M. Karp, G. John, Self-assembled prodrugs: an enzymatically triggered drug-delivery platform, Biomaterials 30 (2009) 383-393.

[4] Y.-h. Yang, H. Aloysius, D. Inoyama, Y. Chen, L.-q. Hu, Enzyme-mediated hydrolytic activation of prodrugs, Acta Pharm. Sinic. B 1 (2011) 143-159.

[5] C. Bao, M. Jin, B. Li, Y. Xu, J. Jina, L. Zhu, Long conjugated 2-nitrobenzyl derivative caged anticancer prodrugs with visible light regulated release: preparation and functionalizations, Org. Biomol. Chem. 10 (2012) 5238-5244.

[6] A.M.L. Hossion, M. Bio, G. Nkepang, S.G. Awuah, Y. You, Visible light controlled release of anticancer drug through double activation of prodrug, ACS Med. Chem. Lett. 4 (2013), 124-127.

[7] B.S. Howerton, D.K. Heidary, E.C. Glazer, Strained ruthenium complexes are potent lightactivated anticancer agents, J. Am. Chem. Soc. 134 (2012) 8324-8327.

[8] Q. Jin, F. Mitschang, S. Agarwal, Biocompatible drug delivery system for photo-triggered controlled release of 5-fluorouracil, Biomacromolecules 12 (2011) 3684-3691.

[9] T. Kurita-Ochiai, Effects of butyric acid on the periodontal tissue, Jpn. Dent. Sci. Rev. 45 (2009), 75-82.

[10] P. Klan, T. Solomek, C.G. Bochet, A. Blanc, R. Givens, M. Rubina, V. Popik, A. Kostikov, J. Wirz, Photoremovable protecting groups in chemistry and biology: reaction mechanisms and efficacy, Chem. Rev. 113 (2013) 119-191.

[11] G. Leriche, L. Chisholm, A. Wagner, Cleavable linkers in chemical biology, Bioorg. Med. Chem. 20 (2012) 571-582.

[12] H. Zhao, E. S. Sterner, E. B. Coughlin, P. Theato, o-Nitrobenzyl alcohol derivatives: opportunities in polymer and materials science, Macromolecules 45 (2012), 1723-1736.

[13] G. Mayer, A. Heckel, Biologically active molecules with a "light switch". Angew. Chem., Int. Ed. 45 (2006) 4900-4921.

[14] V. Hagen, F. Kilic, J. Schaal, B. Dekowski, R. Schmidt, N. Kotzu, [8[Bis(carboxymethyl)aminomethyl]-6-bromo-7-hydroxycoumarin-4-yl]methyl moieties as photoremovable protecting groups for compounds with $\mathrm{COOH}, \mathrm{NH}_{2}, \mathrm{OH}$, and $\mathrm{C}=\mathrm{O}$ functions. $\mathrm{J}$. Org. Chem. 75 (2010) 2790-2797. 
[15] R.S. Givens, M. Rubina, J. Wirz, Applications of $p$-hydroxyphenacyl ( $p \mathrm{HP})$ and coumarin-4ylmethyl photoremovable protecting groups, J. Photochem. Photobiol. Sci. 11 (2012) 472-488.

[16] A.M. Piloto, G. Hungerford, S.P.G. Costa, M.S.T. Gonçalves, Acridinyl methyl esters as photoactive precursors in the release of neurotransmitter amino acids, Photochem. Photobiol. Sci. 12 (2013) 339-347.

[17] A.M.S. Soares, S.P.G. Costa, M.S.T. Gonçalves, Oxazole light triggered protecting groups: synthesis and photolysis of fused heteroaromatic conjugates, Tetrahedron 66 (2010) 8189-8195.

[18] M.J.G. Fernandes, S.P.G. Costa, M.S.T. Gonçalves, Phototriggering of neuroactive amino acids from 5,6-benzocoumarinyl conjugates, Tetrahedron 67 (2011) 2422-2426.

[19] A.M. Piloto, A.M.S. Soares, G. Hungerford, S.P.G. Costa, M.S.T. Gonçalves, Long-wavelength photolysis of amino acid ester derivatives based on 4-methyl-6-methoxy-2-oxo-2H-naphtho[1,2b]pyrans, Eur. J. Org. Chem. (2011) 5447-5451.

[20] A.M.S. Soares, A.M. Piloto, G. Hungerford, S.P.G. Costa, M.S.T. Gonçalves, Photolytic release of butyric acid from oxygen and nitrogen based heteroaromatic cages, Eur. J. Org. Chem. (2012) 922-930.

[21] A.S.C. Fonseca, M.S.T. Gonçalves, S.P.G. Costa, A photoactivable amino acid based on a novel functional coumarin-6-yl-alanine, Amino Acids 43 (2012) 2329-2338.

[22] A.S.C. Fonseca, M.S.T. Gonçalves, S.P.G. Costa, Phenacyl ester derivatives bearing heterocycles as models for photocleavable linkers: synthesis and photolysis studies, Tetrahedron 68 (2012) 8024-8032.

[23] A.S.C. Fonseca, A.M.S. Soares, M.S.T. Gonçalves, S.P.G. Costa, Thionated coumarins and quinolones in the light triggered release of a model amino acid: synthesis and photolysis studies. Tetrahedron 68 (2012) 7892-7900.

[24] A.M. Piloto, A.M.S. Soares, S.P.G. Costa, M.S.T. Gonçalves, Photorelease of amino acids from novel thioxobenzo[f]benzopyran ester conjugates, Amino Acids 42 (2012) 2275-2282.

[25] A. M. Piloto, S. P. G. Costa, M. S. T. Gonçalves, A naphtho[2,1-b]furan as a new fluorescent label: synthesis and spectral characterization, Tetrahedron Lett. 46 (2005) 4757-4760.

[26] A. M. Piloto, G. Hungerford, S. P. G. Costa, M. S. T. Gonçalves, Photoinduced release of neurotransmitter amino acids from novel coumarin fused julolidine ester cages, Eur. J. Org. Chem. (2013) 7715-7723.

[27] M. Göppert-Meyer, Über Elementarakte mit zwei Quantensprüngen, Ann. Phys. 9 (1931) 273295. 
[28] G. Hungerford, L. Ryderfors, M. J. G. Fernandes, M. S. T. Gonçalves, S. P. G. Costa, One- and two-photon time-resolved fluorescence study of neurotransmitter amino acid-5,6-benzocoumarin conjugates, J. Photochem. Photobiol. A: Chem. 215 (2010) 214-222.

[29] M. Jesberger, T.P. Davis, L. Barner, Applications of Lawesson's reagent in organic and organometallic syntheses, Synthesis 13 (2003) 1929-1958.

[30] J. V. Morris, M. A. Mahaney, J. R. Huber, Fluorescence quantum yield determinations. 9,10Diphenylanthracene as a reference standard in different solvents, J. Phys. Chem. 80 (1976) 969-974.

[31] L. Montalti, A. Credi, T. Prodi, M. T. Gandolfi (2006) In: Handbook of Photochemistry. Taylor and Francis, Boca Raton.

[32] C. Muller, P. Even, M.-L. Viriot, M.-C. Carré, Protection and labeling of thymidine by a fluorescent photolabile group, Helv. Chim. Acta 84 (2001) 3735-3741.

[33] M. Drobizhev, N. S. Makarov, S. E. Tillo, T. E. Hughes, A. Rebane, Two-photon absorption properties of fluorescent proteins, Nature Methods 8 (2011) 393-399.

[34] N. S. Makarov, M. Drobizhev, A. Rebane, Two-photon absorption standards in the 550-1600 nm excitation wavelength range, Optics Express 16 (2008) 4029-4047.

[35] D. A. Oulianov, I. V. Tomov, A. S. Dvornikov, P. M. Rentzepis, Observations on the measurement of two-photon absorption cross-section, Optics Commun. 191 (2001) 235-243.

[36] M. A. Albota, C. Xu, W. W. Webb, Two-photon fluorescence excitation cross sections of biomolecular probes from 690 to 960nm, App. Optics 37 (1998) 7352-7356. 


\section{CAPTIONS}

Figure 1. Partial ${ }^{1} \mathrm{H}$ NMR spectra in acetonitrile- $d_{3} / \mathrm{D}_{2} \mathrm{O}(80: 20)$ of the photolysis of $3 H$-benzopyran fused julolidine $12\left(\mathrm{C}=1.17 \times 10^{-2} \mathrm{M}\right)$ at $419 \mathrm{~nm}$ : (a) before irradiation; (b) after irradiation for $4 \mathrm{~h}$; (c) after irradiation for $10 \mathrm{~h}$; (d) butyric acid.

Figure 2. (a) Plot of intensity against power, showing the data for compound $\mathbf{1 1}$ and the slopes calculated for all the compounds. The values obtained (error within 15\%) close to 2 showed a quadratic dependency and are demonstrative of a two-photon process. (b) Fluorescence decay (from 10) showing the instrumental response, decay, fitted function ( 3 exponential) and weighted residuals. Note that the pulse selection process has left the fundamental $(80 \mathrm{MHz})$ train suppressed to a level $\sim 1 \%$ that of the selected pulse.

Figure 3. Plot of the two-photon excitation cross-sections versus wavelength for the different compounds.

Table 1. Yields, UV/visible absorption and fluorescence data for compounds 7-15 in absolute ethanol, methanol/HEPES buffer (80:20) and acetonitrile/HEPES buffer (80:20) solutions.

${ }^{\mathrm{a}}$ in $\mathrm{nm} .{ }^{\mathrm{b}}$ Data in ethanol and methanol/HEPES buffer (80:20) solution was previously reported [20].

${ }^{\mathrm{c}}$ The excitation was carried out at $326 \mathrm{~nm}$.

Table 2. Irradiation times ( $t_{\text {irr }}$ in min), and photochemical quantum yields $\left(\Phi_{\text {Phot }}, \times 10^{-3}\right)$ for the photolysis of conjugates 7-15 at 254, 300, 350 and $419 \mathrm{~nm}$ in methanol/HEPES buffer (80:20) and acetonitrile/HEPES buffer (80:20) solutions. ${ }^{a}$ Data in ethanol and methanol/HEPES buffer (80:20) solution was previously reported [20].

Table 3. Time-resolved fluorescence decay parameters, lifetime $\left(\tau_{\mathrm{i}}\right.$, in ps), and normalised preexponential values $\left(\alpha_{i}\right)$, calculated average lifetime and goodness of fit $\left(\chi^{2}\right)$, for compounds 8-15 in acetonitrile/HEPES buffer (80:20). The excitation wavelength was $378 \mathrm{~nm}$ and the emission wavelengths $\left(\lambda_{\text {em }}\right)$ are also in $\mathrm{nm}$. 
Scheme 1. Synthesis of ester cages of butyric acid 7-15. Reagents and conditions: a) DCC/HOBt, $\mathrm{DMF}, \mathrm{rt}$; $b$ ) KF, DMF, $\mathrm{rt}$; c) Lawesson's reagent, toluene, reflux. 
TABLES

Table 1.

\begin{tabular}{|c|c|c|c|c|c|c|c|c|c|c|c|c|c|c|c|c|}
\hline \multirow{2}{*}{ Compd } & \multirow{2}{*}{$\begin{array}{c}\text { Yield } \\
(\%)\end{array}$} & \multicolumn{5}{|c|}{ Ethanol } & \multicolumn{5}{|c|}{ Methanol/HEPES (80:20) } & \multicolumn{5}{|c|}{ Acetonitrile/HEPES (80:20) } \\
\hline & & $\lambda_{\mathrm{abs}}^{\mathrm{a}}$ & $\log \varepsilon$ & $\lambda_{\mathrm{em}}^{\mathrm{a}}$ & $\Phi_{\mathrm{F}}$ & $\Delta \lambda^{\mathrm{a}}$ & $\lambda_{\mathrm{abs}}^{\mathrm{a}}$ & $\log \varepsilon$ & $\lambda_{\mathrm{em}}{ }^{\mathrm{a}}$ & $\Phi_{\mathrm{F}}$ & $\Delta \lambda^{\mathrm{a}}$ & $\lambda_{\mathrm{abs}}{ }^{\mathrm{a}}$ & $\log \varepsilon$ & $\lambda_{\mathrm{em}}{ }^{\mathrm{a}}$ & $\Phi_{\mathrm{F}}$ & $\Delta \lambda^{\mathrm{a}}$ \\
\hline 7 & 29 & 256 & 3.77 & 303 & 0.08 & 47 & 259 & 3.73 & 304 & 0.07 & 45 & 260 & 3.74 & 304 & 0.13 & 44 \\
\hline 8 & 69 & 360 & 3.71 & 410 & 0.34 & 50 & 360 & 4.12 & 427 & 0.32 & 67 & 360 & 4.15 & 427 & 0.27 & 67 \\
\hline 9 & 70 & 344 & 3.54 & 464 & 0.18 & 120 & 343 & 3.93 & 479 & 0.21 & 136 & 343 & 3.96 & 472 & 0.20 & 129 \\
\hline 10 & 71 & $\begin{array}{l}316 \\
365\end{array}$ & $\begin{array}{l}4.07 \\
3.85\end{array}$ & 459 & 0.53 & 94 & $\begin{array}{l}316 \\
366\end{array}$ & $\begin{array}{l}3.99 \\
3.80\end{array}$ & 470 & 0.49 & 104 & $\begin{array}{l}314 \\
364\end{array}$ & $\begin{array}{l}4.18, \\
3.97\end{array}$ & 464 & 0.45 & 100 \\
\hline $11^{\mathrm{b}}$ & 80 & 350 & 3.92 & 466 & 0.45 & 116 & 352 & 4.22 & 484 & 0.46 & 132 & 345 & 3.86 & 474 & 0.40 & 129 \\
\hline 12 & 34 & 394 & 4.28 & 485 & 0.19 & 91 & 399 & 4.13 & 497 & 0.16 & 98 & 397 & 4.06 & 494 & 0.18 & 97 \\
\hline 13 & 5 & $\begin{array}{l}310, \\
404\end{array}$ & $\begin{array}{l}3.83, \\
3.70\end{array}$ & 447 & 0.03 & 43 & $\begin{array}{l}308 \\
406\end{array}$ & $\begin{array}{l}3.94 \\
3.86\end{array}$ & 448 & 0.03 & 42 & $\begin{array}{l}308 \\
405\end{array}$ & $\begin{array}{l}4.04, \\
3.95\end{array}$ & 437 & 0.03 & 32 \\
\hline $14^{\mathrm{c}}$ & 80 & $\begin{array}{l}283 \\
326 \\
431\end{array}$ & $\begin{array}{l}4.19 \\
4.28 \\
4.21\end{array}$ & 464 & 0.06 & 138 & $\begin{array}{l}326 \\
428\end{array}$ & $\begin{array}{l}4.15 \\
4.12\end{array}$ & 477 & 0.05 & 151 & $\begin{array}{l}326 \\
430\end{array}$ & $\begin{array}{l}4.26, \\
4.23\end{array}$ & 466 & 0.01 & 140 \\
\hline 15 & 70 & 407 & 4.20 & 462 & 0.002 & 55 & 412 & 3.97 & 470 & 0.001 & 58 & 412 & 4.21 & 470 & 0.001 & 58 \\
\hline
\end{tabular}


Table 2.

\begin{tabular}{|c|c|c|c|c|c|c|c|c|c|c|c|c|c|c|c|c|}
\hline \multirow{3}{*}{ Compound } & \multicolumn{8}{|c|}{ Methanol/HEPES (80:20) } & \multicolumn{8}{|c|}{ Acetonitrile/HEPES (80:20) } \\
\hline & \multicolumn{2}{|c|}{$254 \mathrm{~nm}$} & \multicolumn{2}{|c|}{$300 \mathrm{~nm}$} & \multicolumn{2}{|c|}{$350 \mathrm{~nm}$} & \multicolumn{2}{|c|}{$419 \mathrm{~nm}$} & \multicolumn{2}{|c|}{$254 \mathrm{~nm}$} & \multicolumn{2}{|c|}{$300 \mathrm{~nm}$} & \multicolumn{2}{|c|}{$350 \mathrm{~nm}$} & \multicolumn{2}{|c|}{$419 \mathrm{~nm}$} \\
\hline & $t_{\text {irr }}$ & $\Phi_{\text {Phot }}$ & $t_{\text {irr }}$ & $\Phi_{\text {Phot }}$ & $t_{\text {irr }}$ & $\Phi_{\text {Phot }}$ & $t_{\text {irr }}$ & $\Phi_{\text {Phot }}$ & $t_{\text {irr }}$ & $\Phi_{\text {Phot }}$ & $t_{\text {irr }}$ & $\Phi_{\text {Phot }}$ & $t_{\text {irr }}$ & $\Phi_{\text {Phot }}$ & $t_{\text {irr }}$ & $\Phi_{\text {Phot }}$ \\
\hline 7 & 11 & 1.71 & 26 & 0.37 & 76 & 0.12 & 2217 & 0.004 & 14 & 1.49 & 41 & 0.225 & 106 & 0.085 & 1198 & 0.008 \\
\hline 8 & 4 & 1.88 & 7 & 0.60 & 2 & 1.64 & 83 & 0.044 & 58 & 0.129 & 120 & 0.03 & 328 & 0.011 & 2499 & 0.001 \\
\hline 9 & 192 & 0.063 & 230 & 0.027 & 182 & 0.033 & 5985 & 0.001 & 186 & 0.062 & 184 & 0.033 & 172 & 0.031 & 4288 & 0.001 \\
\hline 10 & 432 & 0.039 & 546 & 0.015 & 555 & 0.014 & 3324 & 0.0024 & 457 & 0.025 & 481 & 0.012 & 493 & 0.012 & 9989 & 0.001 \\
\hline $11^{\mathrm{a}}$ & 178 & 0.034 & 253 & 0.011 & 263 & 0.011 & 490 & 0.006 & 278 & 0.054 & 294 & 0.024 & 270 & 0.026 & 520 & 0.013 \\
\hline 12 & 6 & 1.41 & 9 & 0.426 & 6 & 0.79 & 5 & 0.790 & 5 & 1.87 & 7 & 0.691 & 4 & 1.04 & 4 & 1.03 \\
\hline 13 & 55 & 0.262 & 178 & 0.039 & 210 & 0.032 & 142 & 0.047 & 53 & 0.202 & 129 & 0.044 & 292 & 0.018 & 193 & 0.029 \\
\hline 14 & 212 & 0.040 & 371 & 0.001 & 526 & 0.007 & 351 & 0.010 & 153 & 0.042 & 585 & 0.005 & 308 & 0.010 & 296 & 0.010 \\
\hline 15 & 90 & 0.129 & 184 & 0.029 & 296 & 0.018 & 98 & 0.129 & 57 & 0.114 & 111 & 0.020 & 203 & 0.023 & 76 & 0.040 \\
\hline
\end{tabular}


Table 3.

\begin{tabular}{cccccccccc}
\hline compound & $\lambda_{\text {em }}$ & $\tau_{1}$ & $\tau_{2}$ & $\tau_{3}$ & $\alpha_{1}$ & $\alpha_{2}$ & $\alpha_{3}$ & $\tau_{\text {ave }}$ & $\chi^{2}$ \\
\hline $\mathbf{8}$ & 410 & $511 \pm 60$ & $10791 \pm 27$ & & 0.31 & 0.69 & & 7655 & 0.98 \\
$\mathbf{9}$ & 475 & $113 \pm 39$ & $2631 \pm 507$ & $3591 \pm 75$ & 0.24 & 0.34 & 0.42 & 2416 & 1.14 \\
$\mathbf{1 0}$ & 460 & $155 \pm 150$ & $4954 \pm 174$ & $10379 \pm 67$ & 0.13 & 0.53 & 0.34 & 6121 & 1.08 \\
$\mathbf{1 1}$ & 470 & $65 \pm 39$ & $3522 \pm 543$ & $7208 \pm 33$ & 0.38 & 0.09 & 0.53 & 4142 & 1.09 \\
$\mathbf{1 2}$ & 470 & $240 \pm 30$ & $2241 \pm 99$ & $5108 \pm 60$ & 0.46 & 0.35 & 0.19 & 1835 & 1.20 \\
$\mathbf{1 3}$ & 470 & $216 \pm 54$ & $2991 \pm 75$ & $5831 \pm 175$ & 0.28 & 0.64 & 0.08 & 2434 & 1.16 \\
$\mathbf{1 4}$ & 470 & $91 \pm 39$ & $4812 \pm 162$ & $10065 \pm 93$ & 0.42 & 0.35 & 0.23 & 4100 & 1.08 \\
$\mathbf{1 5}$ & 470 & $118 \pm 129$ & $2445 \pm 339$ & $6893 \pm 24$ & 0.25 & 0.11 & 0.64 & 4701 & 1.10 \\
\hline
\end{tabular}




\section{FIGURES}

Figure 1.

-O-CO- $\mathrm{CH}_{2}-\mathrm{CH}_{2}-\mathrm{CH}_{3}$

$\mathrm{CH}_{2}$ Het

d)

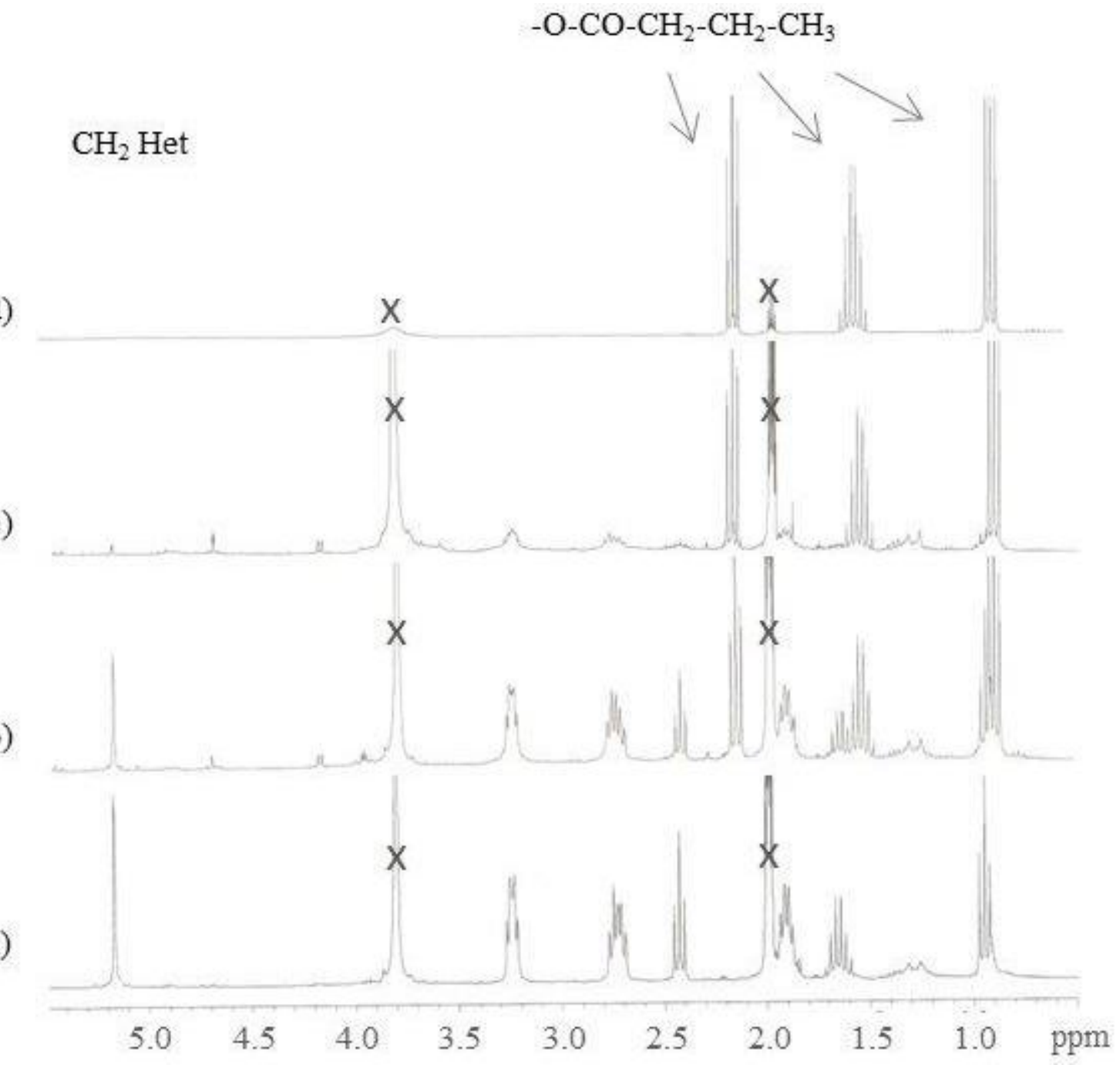


Figure 2.
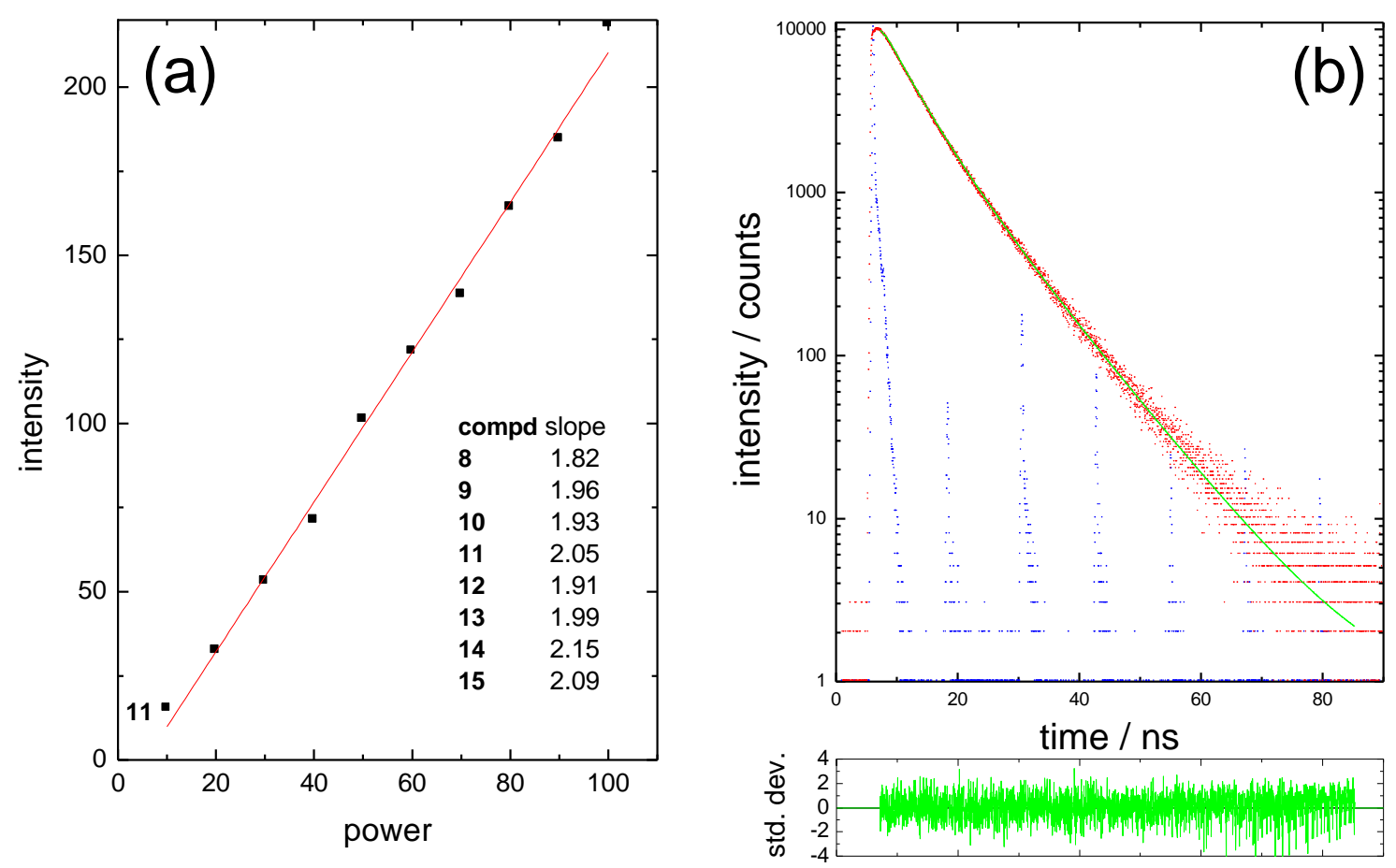
Figure 3.

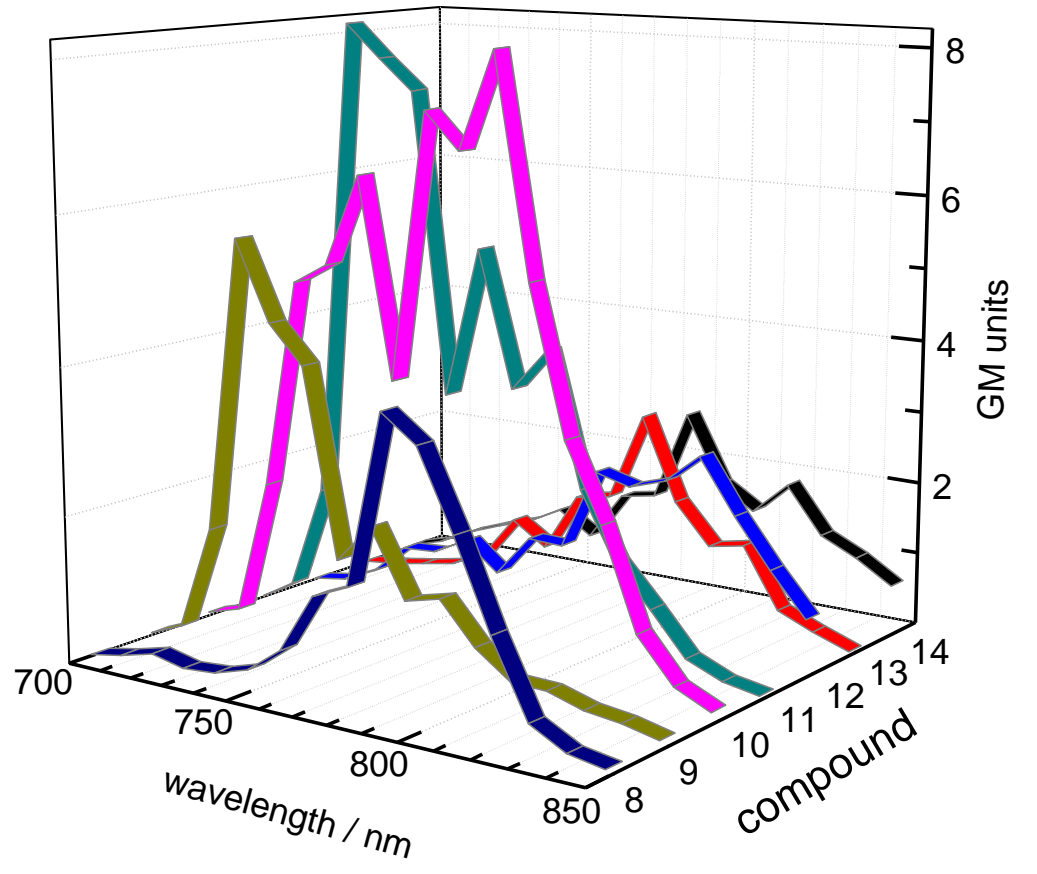




\section{SCHEMES}

Scheme 1.
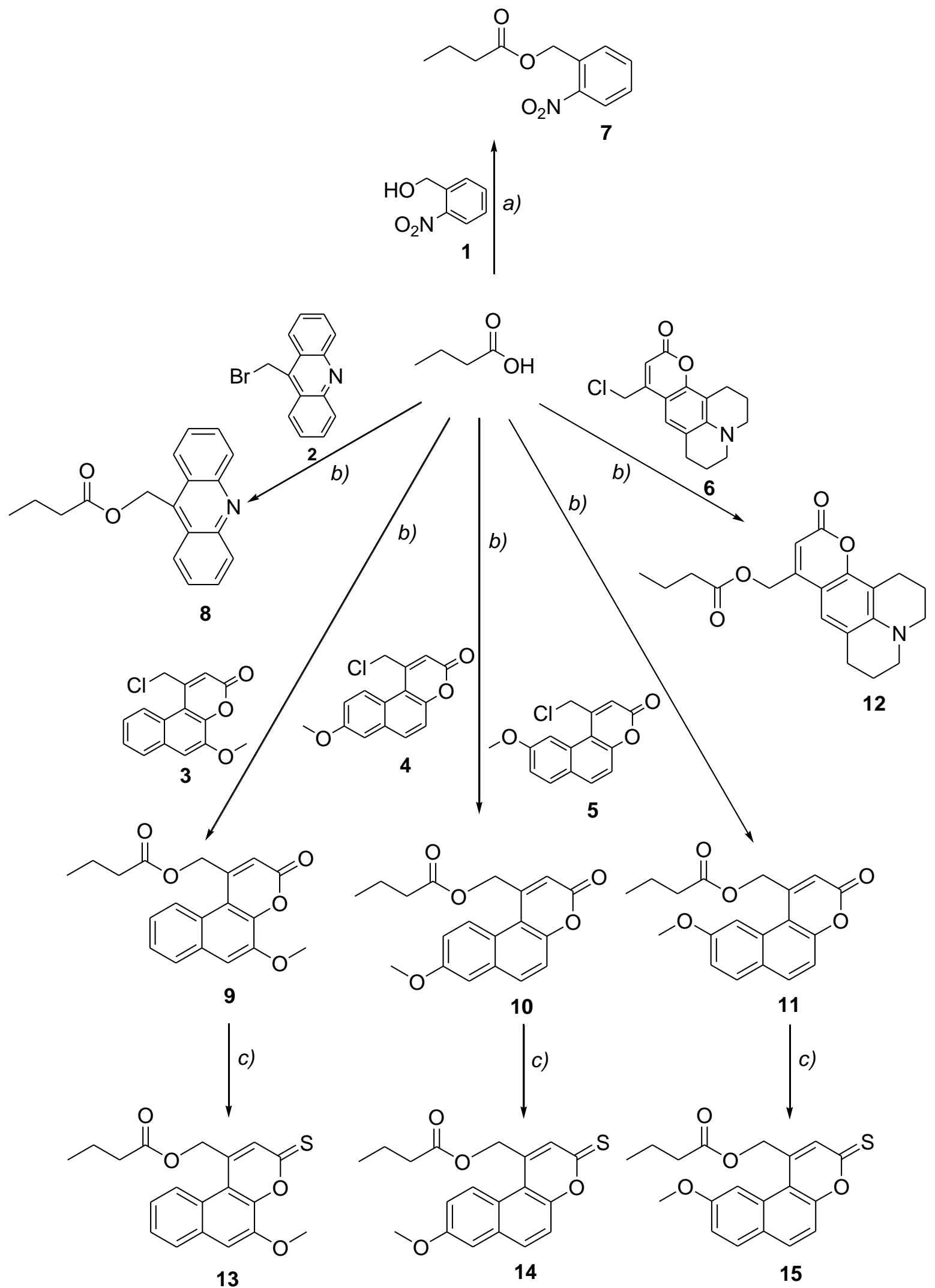